\title{
Sulfopin, a selective covalent inhibitor of Pin1, blocks Myc-driven tumor initiation and growth in vivo
}

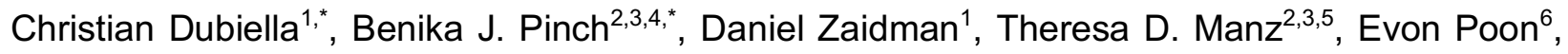
Shuning $\mathrm{He}^{7}$, Efrat Resnick ${ }^{1}$, Ellen M. Langer ${ }^{8,9}$, Colin J. Daniel ${ }^{8,9}$, Hyuk-Soo Seo ${ }^{2}$, Ying Chen ${ }^{10}$, Scott B. Ficarro ${ }^{2,11,12,13}$, Yann Jamin ${ }^{14}$, Xiaolan Lian ${ }^{15,16,17}$, Shin Kibe ${ }^{15,16,17}$, Shingo Kozono ${ }^{15,16,17}$, Kazuhiro Koikawa ${ }^{15,16,17}$, Zainab M. Doctor ${ }^{2,3}$, Behnam Nabet ${ }^{2,3}$, Christopher M. Browne ${ }^{2,3,18}$, Annan Yang ${ }^{2,19}$, Liat Stoler-Barak ${ }^{20}$, Richa B. Shah ${ }^{21,22}$, Nick E. Vangos ${ }^{2}$, Ezekiel A. Geffken ${ }^{2}$, Roni Oren ${ }^{23}$, Samuel Sidi ${ }^{21,22}$, Ziv Shulman ${ }^{20}$, Chu Wang ${ }^{10}$, Jarrod A. Marto ${ }^{2,11,12,13}$, Sirano DhePaganon $^{2}$, Thomas Look ${ }^{7,24}$, Xiao Zhen Zhou ${ }^{15,16,17}$, Kun Ping Lu ${ }^{15,16,17}$, Rosalie C. Sears ${ }^{8,9,25}$, Louis Chesler ${ }^{6}$, Nathanael S. Gray ${ }^{2,3, \#}$, Nir London ${ }^{1, \#}$

1 Department of Organic Chemistry, The Weizmann Institute of Science, Rehovot, 7610001, Israel.

2 Department of Cancer Biology, Dana-Farber Cancer Institute, Boston, MA 02115, USA.

${ }^{3}$ Department of Biological Chemistry and Molecular Pharmacology, Harvard Medical School, Boston, MA 02215, USA.

${ }^{4}$ Department of Chemistry and Chemical Biology; Department of Chemical Biology, Harvard University, Cambridge, MA, 02138, USA.

${ }^{5}$ Department of Pharmaceutical and Medicinal Chemistry, Saarland University, Saarbruecken, Germany.

${ }^{6}$ Division of Clinical Studies, The Institute of Cancer Research, London, UK.

${ }^{7}$ Department of Pediatric Oncology, Dana-Farber Cancer Institute, Harvard Medical School, Boston, MA, 02115, USA.

${ }^{8}$ Department of Molecular and Medical Genetics, Oregon Health \& Science University, Portland, Oregon.

${ }^{9}$ Knight Cancer Institute, Oregon Health \& Science University, Portland, Oregon.

${ }^{10}$ College of Chemistry and Molecular Engineering, Peking University, Beijing, 100871, China.

${ }^{11}$ Department of Oncologic Pathology, Dana-Farber Cancer Institute, Harvard Medical School, Boston, MA, 02115, USA.

12 Blais Proteomics Center, Dana-Farber Cancer Institute, Harvard Medical School, Boston, MA, 02115, USA.

${ }^{13}$ Department of Pathology, Brigham and Women's Hospital and Harvard Medical School, Boston, MA, 02115, USA.

${ }^{14}$ Division of Radiotherapy and Imaging, The Institute of Cancer Research, London, UK.

${ }^{15}$ Department of Medicine, Division of Translational Therapeutics, Beth Israel Deaconess Medical Center, Harvard Medical School, Boston, MA 02215, USA.

${ }^{16}$ Cancer Research Institute, Beth Israel Deaconess Medical Center, Harvard Medical School, Boston, MA 02215, USA.

${ }^{17}$ Broad Institute of MIT and Harvard, Cambridge, MA 02142, USA.

${ }^{18}$ Currently at: Discovery Biology, Discovery Sciences, Biopharmaceuticals R\&D, AstraZeneca, Boston, MA USA

${ }^{19}$ Department of Medical Oncology, Dana-Farber Cancer Institute, Boston, MA 02115, USA.

${ }^{20}$ Department of Immunology, The Weizmann Institute of Science, Rehovot, 7610001, Israel.

${ }^{21}$ Department of Medicine, Division of Hematology and Medical Oncology, Tisch Cancer Institute, Icahn School of Medicine at Mount Sinai, New York, NY, USA

${ }_{22}^{2}$ Department of Cell, Developmental and Regenerative Biology, The Graduate School of Biomedical Sciences, Icahn School of Medicine at Mount Sinai, New York, NY, USA

${ }^{23}$ Department of Veterinary Resources, The Weizmann Institute of Science, Rehovot, 7610001, Israel.

${ }^{24}$ Division of Pediatric Hematology/Oncology Boston Children's Hospital, Boston, MA, 02115, USA.

${ }^{25}$ Brenden-Colson Center for Pancreatic Care, Oregon Health \& Science University, Portland, Oregon.

* equal contribution.

\# e-mail: nir.london@weizmann.ac.il; nathanael_gray@dfci.harvard.edu

Keywords: covalent fragments; covalent probes; Pin1; neuroblastoma; Myc 


\begin{abstract}
The peptidyl-prolyl cis-trans isomerase, Pin1, acts as a unified signaling hub that is exploited in cancer to activate oncogenes and inactivate tumor suppressors, in particular through up-regulation of c-Myc target genes. However, despite considerable efforts, Pin1 has remained an elusive drug target. Here, we screened an electrophilic fragment library to discover covalent inhibitors targeting Pin1's active site nucleophile - Cys113, leading to the development of Sulfopin, a double-digit nanomolar Pin1 inhibitor. Sulfopin is highly selective for Pin1, as validated by two independent chemoproteomics methods, achieves potent cellular and in vivo target engagement, and phenocopies genetic knockout of Pin1. Although Pin1 inhibition had a modest effect on viability in cancer cell cultures, Sulfopin induced downregulation of c-Myc target genes and reduced tumor initiation and tumor progression in murine and zebrafish models of MYCN-driven neuroblastoma. Our results suggest that Sulfopin is a suitable chemical probe for assessing Pin1dependent pharmacology in cells and in vivo. Moreover, these studies indicate that Pin1 should be further investigated as a potential cancer target.
\end{abstract}




\section{Introduction}

Cancer relies on multiple signaling pathways to sustain proliferation and downregulate apoptotic signals ${ }^{1}$, including the phosphorylation of serine/threonine - proline motif (pSer/ThrPro) ${ }^{2,3}$ found in many cellular proteins. This motif is specifically recognized and isomerized by the peptidyl-prolyl isomerase NIMA-interacting-1 (Pin1), which is the only known phosphorylationdependent isomerase amongst the $\sim 30$ peptidyl-prolyl cis-trans isomerases (PPlases) in the human proteome ${ }^{4}$. Pin1-mediated isomerization was shown to impact substrate stability ${ }^{5-9}$, activity ${ }^{10,11}$, subcellular localization ${ }^{8}$, and binding to interaction partners, including Prolinedirected kinases and phosphatases, which are mostly trans-specific ${ }^{12-14}$. Thus, Pin1 represents a unified signaling hub that is exploited by cancer to activate oncogenes and inactivate tumor suppressors ${ }^{15,16}$.

Several lines of evidence suggest that aberrant Pin1 activation drives oncogenesis. Pin1 is over-expressed and/or -activated in at least 38 tumor types ${ }^{17}$. While elevated Pin1 expression correlates with poor clinical prognosis ${ }^{18,19}$, polymorphisms that lower Pin1 expression are associated with reduced cancer risk ${ }^{20}$. Pin1 sustains proliferative signaling in cancer cells by upregulating over 50 oncogenes or growth-promoting factors ${ }^{15,16}$, including NF-kB ${ }^{9}$, Notch1 ${ }^{21}$ and $\mathrm{C}-\mathrm{Myc}^{22}$, while suppressing over 20 tumor suppressors or growth-inhibiting factors, such as FOXOs ${ }^{23}, \mathrm{Bcl} 2{ }^{24}$ and RARa ${ }^{25}$. Furthermore, Pin1-null mice are resistant to tumorigenesis induced by mutant p53 ${ }^{26}$, activated HER2/RAS ${ }^{27}$, or constitutively expressed c-Myc ${ }^{28}$. In addition, Pin1 inhibition sensitizes cancer cells to chemotherapeutics ${ }^{25,29,30}$, radiation therapy ${ }^{31}$ and blocks the tumorigenesis of cancer stem cells ${ }^{16,32,33}$. However, as evidenced by the Cancer Dependency Map ${ }^{34}$, Pin1 is not essential to cellular viability and Pin1-null mice are viable, though they develop premature aging phenotypes ${ }^{7,35}$.

Taken together, these studies suggest that pharmacological inhibition of Pin1 has the potential to block multiple cancer-driving pathways simultaneously ${ }^{16}$ and with limited toxicity. Indeed, compounds that inhibit Pin1, such as juglone ${ }^{36}$, all-trans retinoic acid (ATRA) ${ }^{37}$, arsenic trioxide (ATO) ${ }^{38}$ and KPT-6566 ${ }^{39}$, exhibit anti-cancer activity and have been used to investigate the role of Pin1 in oncogenesis. Nevertheless, these compounds have been shown to lack specificity and/or cell permeability thus making them unreliable as tools to interrogate the consequences of pharmacological inhibition of Pin1 in vivo ${ }^{40-42}$. We have recently developed a selective Pin1 covalent peptide inhibitor ${ }^{43}$. However, it too, was unsuitable for in vivo applications.

The active-site of Pin1 contains a nucleophilic cysteine residue (Cys113) which is suitable for the development of targeted covalent inhibitors ${ }^{43-45}$. Such covalent inhibitors have several 
advantages over non-covalent inhibitors ${ }^{46-48}$ and have been extremely successful against both traditional $^{49-51}$ and challenging targets ${ }^{52-55}$.

To explore this strategy, we undertook a covalent Fragment-Based Drug Discovery (FBDD) screening campaign targeting Cys113 in Pin1's PPlase active site. FBDD, which focuses on low molecular weight compounds (typically < $300 \mathrm{Da}$ ), is a successful hit discovery approach ${ }^{56-58}$ that has led to several drugs and chemical probes ${ }^{57,59}$. It offers good coverage of chemical space and a high probability of binding due to lower molecular complexity ${ }^{60,61}$. Covalent FBDD, which increasingly comes to the fore ${ }^{62-72}$, combines the advantages of FBDD with the improved potency conferred by covalent bond formation.

Optimization of screening hits from this campaign, led to the development of Sulfopin, a double-digit nanomolar, highly selective Pin1 inhibitor that engages Pin1 in cells and in vivo. We found that Pin1 inhibition induced modest viability effects in 2D cancer cell culture only after prolonged exposure, and resulted in the downregulation of Myc-dependent target genes. In MYCN-driven models of neuroblastoma, both in zebrafish and in mice, Sulfopin significantly reduced tumor initiation as well as tumor progression. Sulfopin is therefore the first selective Pin1 inhibitor suitable for the evaluation of Pin1 biology in vivo, and provides evidence that Pin1 warrants further exploration as a potential anti-cancer target.

\section{Results}

A covalent fragment screen identifies Pin1 binders

We previously compiled a library of 993 electrophilic fragments featuring mildly reactive 'warheads' that can react covalently with cysteines in target proteins ${ }^{62}$. We screened our library against Pin1 to identify fragment leads for covalent inhibitor development. Purified catalytic domain of Pin1 was incubated with the fragment library ( $2 \mu \mathrm{M}$ protein, $200 \mu \mathrm{M}$ compound; $24 \mathrm{~h}$ at $4{ }^{\circ} \mathrm{C}$ ), followed by intact protein liquid chromatography/mass-spectrometry (LC/MS) to identify and quantify Pin1 labeling (Fig. 1A). In total, 111 fragments irreversibly labeled Pin1 by $>50 \%$ under the assay conditions (Fig. 1B; Supp. Dataset 1). Among the 48 top hits (labeling > 75\%) nine chloroacetamides shared a common cyclic sulfone core, suggesting a structure-activity relationship (SAR; Fig. 1B, see Supp. Fig. 1 \& Supp. Table 1 for a full list of hits containing this motif). Given that the identified sulfone-containing hits were non-promiscuous in previous fragment screens against a diverse panel of proteins ${ }^{62}$, we selected them for further development. 
To avoid undesired reactivity arising from the presence of an additional Michael acceptor in the 2-sulfolene fragments, we focused exclusively on the sulfolane analogs.

Fragment optimization yields potent Pin 1 binding that is not driven by high warhead reactivity

To guide compound optimization, we used the covalent docking program, DOCKovalent 73. Docking the sulfolane hits into various Pin1 structures and inspecting highly ranked poses, suggested two plausible binding modes, in which the sulfolane or a lipophilic moiety ('R' in Fig. 1B) either protruded into the hydrophobic proline-binding pocket, or interacted with a hydrophobic patch adjacent to Cys113 (Supp. Fig. 2). Both poses suggested maintaining the sulfolane, while diversifying the lipophilic moiety.

To optimize these original hits, we synthesized or purchased a total of 26 sulfolanecontaining compounds, featuring a range of aliphatic, arylic, biphenylic or heterocyclic side-chains (Supp. Fig. 3). To identify high-affinity binders, we assessed their irreversible labeling of Pin1 in a second screen under more stringent conditions, using a 1:1 ratio of protein:compound, and a shorter incubation time ( $2 \mu \mathrm{M}$ compound; $1 \mathrm{~h}$ at RT). Remarkably, 25 out of 26 of these secondgeneration compounds showed better labeling than the original screening hits, which showed no labeling under these new conditions (Supp. Table 2). Overall, the hits from this second screen revealed that a wide range of lipophilic moieties were tolerated. We concluded that an additional methylene group between the amide and the lipophilic side chain was crucial for Pin1 labeling, as exemplified by four matched molecular pairs that lacked this group and showed no labeling (Supp. Fig. 4). The top ten binders from the second screen (Fig. 1C,F) showed 35-65\% Pin1 labeling. We next evaluated these analogs in a competitive fluorescence polarization (FP) assay using a FITC-labeled substrate mimetic peptide inhibitor ${ }^{74}$. Following a $14 \mathrm{~h}$ incubation with recombinant full-length Pin1, all analogs competed in the FP to a greater extent than juglone, an often cited Pin1 inhibitor (Fig. 1D).

Identifying and excluding overly reactive and potentially promiscuous compounds is critical in the development of covalent probes. Accordingly, we assessed the thiol reactivity of the top binders using a high-throughput assay that we previously applied to the entire fragment library ${ }^{62}$. We found that there was no correlation between labeling efficiency and reactivity (Fig. 1C; Pearson $R=0.003$; Supp. Fig. 5). This was particularly evident when comparing Pin1-3, which has a tert-butyl side chain, with the structurally similar Pin-1-13, which has a cyclopropyl side chain. Both compounds showed similar Pin1 labeling (48\% and $46 \%)$, but their reactivity varied by an order of magnitude, with Pin1-3 being dramatically less reactive. Furthermore, the 
compound with the highest degree of Pin1 labeling, Pin1-2-3, showed only median reactivity relative to the entire panel.

We have previously shown ${ }^{62}$ that electrophiles with reactivity rate constants higher than $10^{-7} \mathrm{M}^{-1} \mathrm{~s}^{-1}$ may exhibit non-selective cytotoxicity. We evaluated selected compounds from the top Pin1 labelers in a viability assay against IMR90 lung fibroblasts, and Pin1-3 was the only compound that did not show any toxicity up to $25 \mu \mathrm{M}$ (Supp. Table 3). Pin1-3 has the lowest inherent reactivity of the top identified Pin1 labelers, and does not exhibit non-selective cytotoxicity, therefore showing the best balance of potency and selectivity. For this reason, we selected Pin1-3, henceforth Sulfopin, for further evaluation.

A

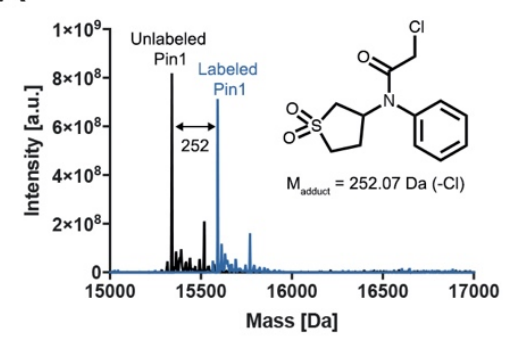

B

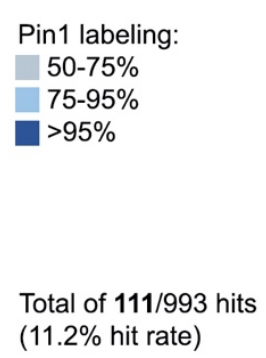

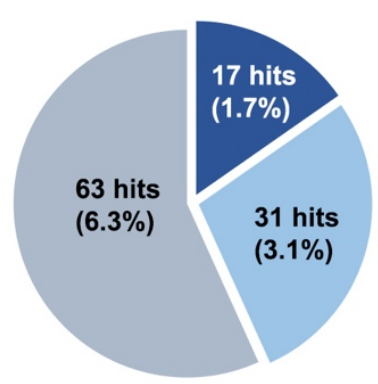

$18.75 \%$ of top hits

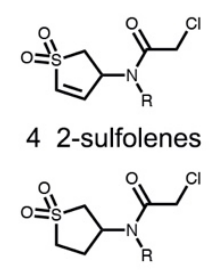

5 sulfolanes

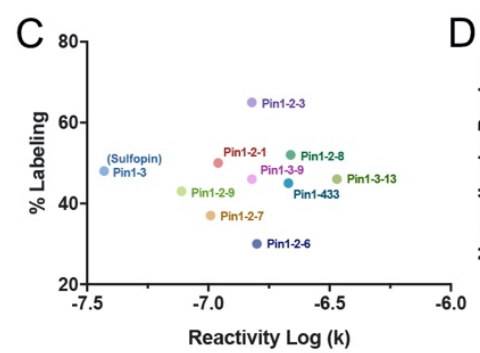

$\mathrm{F}$
D
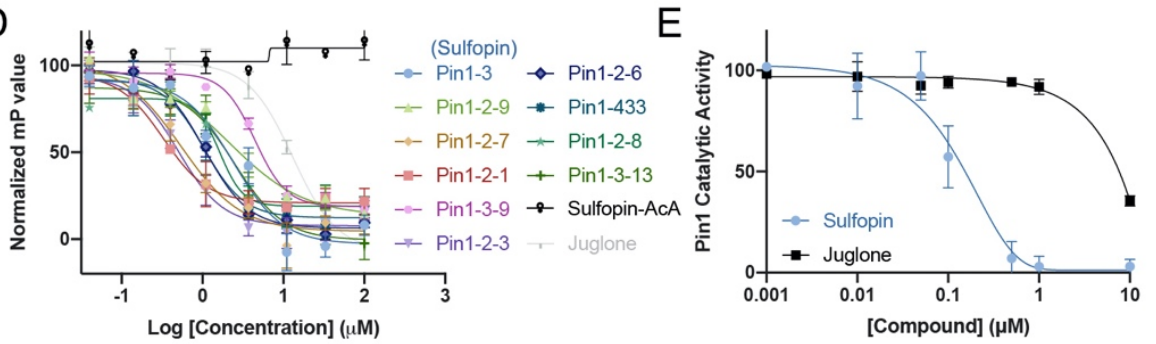

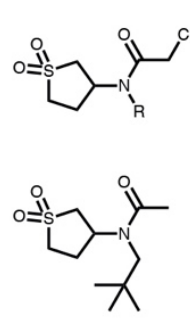

Sulfopin-AcA

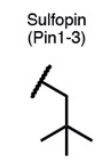

Sultopin<smiles>C1CCCCC1</smiles>

Pin1-3-9<smiles>CCc1ccccc1Cc1ccccc1</smiles>

Pin1-2-7
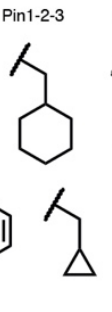

Pin1-3-13
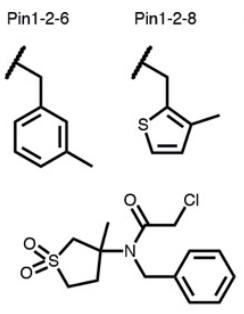

Pin1-2-9
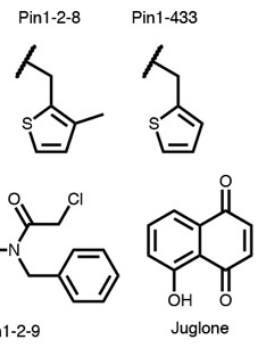

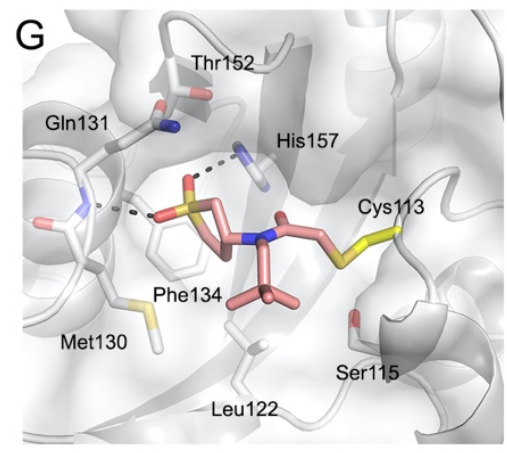

Figure 1. The discovery of a covalent Pin1 binding fragment. A. Intact protein LC/MS spectra of Pin1 (black) directly identifies covalent binders (blue) in the electrophilic library screen $(200 \mu \mathrm{M}$ compound for $24 \mathrm{~h})$ B. Distribution of hits in the Pin1 screening campaign and their corresponding labeling in percent: Nine hits (18.75\%) out of 48 top hits that labeled Pin1 $>75 \%$ (dark and light blue) share sulfolene or sulfolane moieties. C. 2D-analysis of the top ten optimized binders (structures in F.): Labeling $\%$ in the LC/MS-assay is plotted against reactivity $(\log (k))$ suggests Sulfopin for further biological evaluation. D. Fluorescence polarization assay with Pin1 and the top ten binders including juglone and a non-reactive control (Sulfopin-AcA), after $14 \mathrm{~h}$ pre-incubation. See Supp. Table 3 for apparent $\mathrm{K}_{\mathrm{i}} \mathrm{E}$. Substrate activity 
assay of Pin1 with Sulfopin and juglone. F. Structures of the top ten binders in the Pin1 labeling LC/MSassay, the non-reactive control Sulfopin-AcA and juglone. G. X-ray crystal structure of Pin1 in complex with Sulfopin (1.4 Å resolution, PDB code 6VAJ). Pin1 (white) with relevant side-chains in stick representation. Sulfopin is shown in pink. Hydrogen-bonds are depicted as dashed lines.

\section{Sulfopin potently binds and inhibits Pin1}

Sulfopin displayed potent Pin1 binding in the FP assay ${ }^{37}$ with an apparent $\mathrm{K}_{\mathrm{i}}=17 \mathrm{nM}$ (after 14 h; Fig. 2B). A corresponding reversible compound (Sulfopin-AcA; Fig. 1F), which lacks the chloride leaving group, was inactive in the FP assay, suggesting that the binding affinity of Sulfopin is dependent on its electrophile (Fig. 1D,2B). Sulfopin-AcA was subsequently used as a negative control compound. We then performed the FP assay in a dose- and time-dependent manner to determine the $\mathrm{K}_{\text {inact }}$ of Sulfopin to be $0.03 \mathrm{~min}^{-1}$, with a second order rate constant $\left(\mathrm{K}_{\text {inact }} / \mathrm{K}_{\mathrm{i}}\right)$ of $84 \mathrm{M}^{-1} \mathrm{~s}^{-1}$ (Supp. Fig. 6). Sulfopin also inhibited the catalytic activity of Pin1 with an apparent $\mathrm{K}_{\mathrm{i}}$ of $211 \mathrm{nM}$ measured at $12 \mathrm{~h}$, as determined using a chymotrypsin-coupled peptidylprolyl isomerization assay ${ }^{75}$ (PPlase assay; Fig. 1E).

To evaluate the binding mode of Sulfopin, we determined the co-crystal structure of Pin1 in complex with Sulfopin at $1.4 \AA$ resolution (Supp. Table 4). The complex structure shows clear electron density to Cys 113 in the $2 \mathrm{~F}_{\mathrm{O}}-\mathrm{F}_{\mathrm{C}}$ omit map, which confirmed a covalent interaction (Supp. Fig. 7). In this structure, the sulfolane ring occupies the hydrophobic proline-binding pocket that is formed by Met130, Gln131, Phe134, Thr152 and His157 (Fig. 1G). Furthermore, the sulfonyl oxygens mediate hydrogen bonds to the backbone amide of Gln131, and the imidazole $\mathrm{NH}$ of His 157. These hydrogen bonds are analogous to those formed between Pin1 and arsenic trioxide

${ }^{38}$ (Supp. Fig. 8). The tert-butyl group of Sulfopin covers a shallow hydrophobic patch formed by Ser114, Leu122 and Met130, but is mostly solvent-exposed, which explains the broad range of hydrophobic moieties that were tolerated at this position during the optimization campaign.

Despite being a very small ligand (heavy atom count: 17, cLogP: 0.36), Sulfopin efficiently exploits interactions with the active site of Pin1, even in the absence of a negatively charged moiety to interact with the phosphate-binding pocket, thus overcoming the cell permeability issues of previous Pin1 inhibitors, which are often highly anionic ${ }^{74,76-78}$.

\section{Sulfopin engages Pin1 in cells and in vivo}

To evaluate the target engagement of Sulfopin in cells, we developed a desthiobiotin (DTB)-labeled probe for competition pull-down experiments. Based on the co-crystal structure of 
Sulfopin bound to Pin1, we derivatized the mostly solvent-exposed tert-butyl group of Sulfopin with a PEG-linked DTB (Sulfopin-DTB; Fig. 2A). Sulfopin-DTB showed similar potency (apparent $\mathrm{K}_{\mathrm{i}}=38 \mathrm{nM}$ in FP assay; Fig. 2B), and successfully engaged Pin1 in PATU-8988T cell lysates, achieving robust pull-down at $1 \mu \mathrm{M}$ following a $1 \mathrm{~h}$ incubation period (Supp. Fig. 9A).

To assess the cellular target engagement and cell permeability of Sulfopin, we performed a live cell competition assay in PATU-8988T and HCT116 cells. A $1 \mu \mathrm{M}$ treatment of Sulfopin achieved complete Pin1 engagement within $4 \mathrm{~h}$ (with about 50\% after $2 \mathrm{~h}$; Fig. 2C), and maintained significant engagement up to $72 \mathrm{~h}$ (Fig. 2E). Sulfopin exhibited dose-dependent competition for Pin1 binding, with maximal competition evident at 0.5-1 $\mu \mathrm{M}$, while the negative control Sulfopin-AcA showed no competition (Fig. 2D). We found that this cellular engagement was extensible to other cell lines, such as IMR32, and MDA-MB-231 (Supp. Fig. 9C,D).

Ultimately, we were interested whether Sulfopin could be used in vivo. Sulfopin had encouraging metabolic stability in mouse hepatic microsomes $\left(T_{1 / 2}=41 \mathrm{~min}\right)$, prompting us to submit it for pharmacokinetic (PK) profiling. In three mice, oral administration of $10 \mathrm{mg} / \mathrm{kg}$ Sulfopin achieved an average $\mathrm{C}_{\max }$ of $11.5 \mu \mathrm{M}$ and oral bioavailability (F\%) of $30 \%$ (Supp. Table 5), suggesting that Sulfopin is suitable for oral in vivo dosing. We next evaluated the toxicity of Sulfopin in an acute toxicity model in which mice were administered daily doses of 10,20 or 40 $\mathrm{mg} / \mathrm{kg}$ Sulfopin by intraperitoneal (IP) injection for two weeks. No adverse effects or weight loss were recorded, and a post-mortem examination found no readily detectable pathologies.

Using Sulfopin-DTB, we were also able to assess the in vivo engagement of Sulfopin. To do this, mice were treated with three doses (over the course of two days) of vehicle, 10, or 20 $\mathrm{mg} / \mathrm{kg}$ Sulfopin by oral gavage, followed by lysis of the spleens for a competition pull-down experiment. Effective Pin1 engagement was observed in 1 out of 3 mice treated with $10 \mathrm{mg} / \mathrm{kg}$ Sulfopin, and in 3 out of 3 mice treated with $20 \mathrm{mg} / \mathrm{kg}$ Sulfopin, with target engagement monitored by loss of Sulfopin-DTB-mediated pull-down (Fig. 2F). Based on these results, we chose a 40 $\mathrm{mg} / \mathrm{kg}$ dose for further mouse experiments to ensure complete Pin1 engagement. 
A

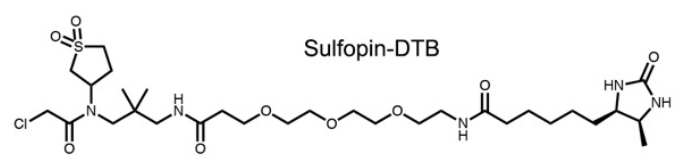

B

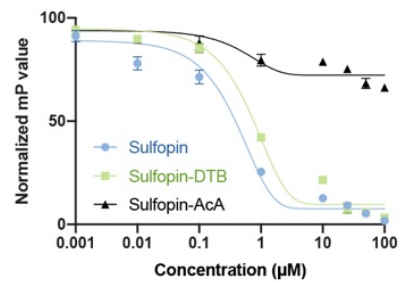

PATU-8988T cells

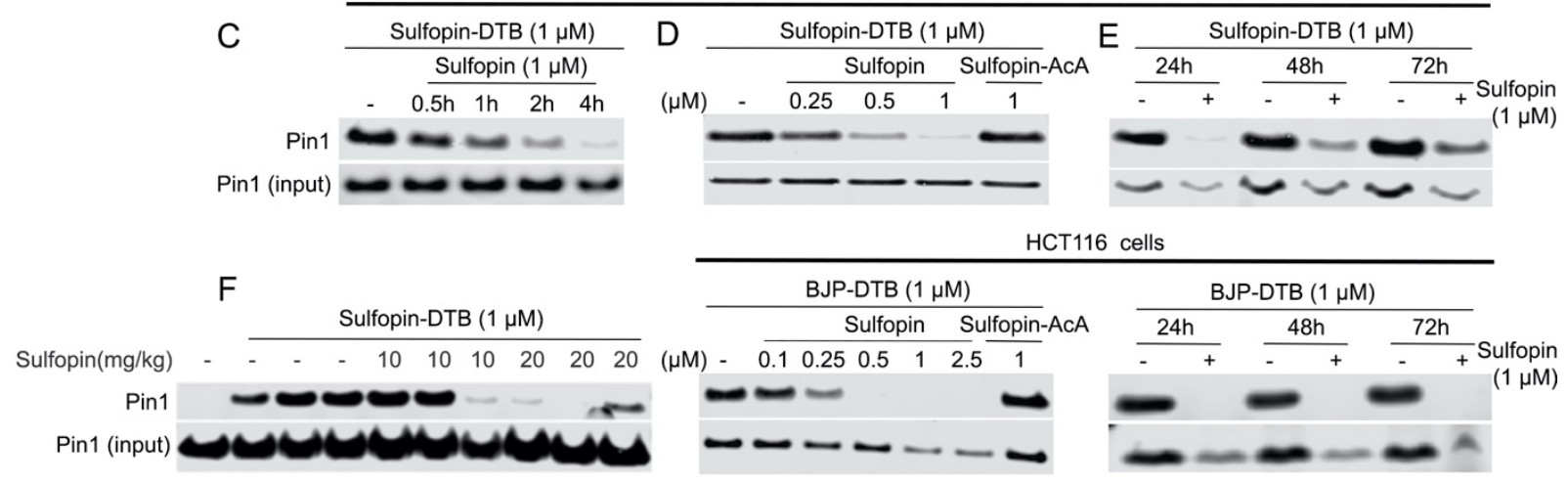

Figure 2. Sulfopin engages Pin1 in cells and in vivo.

A. Chemical structure of the desthiobiotin probe Sulfopin-DTB. B. Competitive FP assay shows that Sulfopin-DTB binds Pin1 with similar potency to Sulfopin. C. Sulfopin shows time dependent engagement in PATU-8988T cells. Cells were incubated for the indicated times with Sulfopin $(1 \mu \mathrm{M})$, then lysed and incubated with Sulfopin-DTB $(1 \mu \mathrm{M})$. Following enrichment, Pin1 levels were analysed by Western blot. D. Sulfopin fully engages Pin1 in PATU-8988T cells at $1 \mu \mathrm{M}$ and in HCT116 cells at $0.5 \mu \mathrm{M}$ (See Supp. Fig. 9B for a structure of BJP-DTB). Cells were incubated with Sulfopin at the indicated concentration for $5 \mathrm{~h}$, followed by lysis and DTB probe incubation $(1 \mathrm{~h}, 1 \mu \mathrm{M})$. The non-covalent control Sulfopin-AcA is not able to engage Pin1. E. Sulfopin shows long-term engagement with Pin1. PATU-8988T and HCT116 cells were incubated with or without Sulfopin for the indicated times, followed by lysis and incubation with DTB probes. Significant engagement (>50-100\%) is still evident after $72 \mathrm{~h}$. F. Sulfopin engages Pin1 in vivo. Mice were treated by oral gavage with the indicated amounts of Pin1, over two days for a total of three doses. Following this treatment, their spleens were lysed for a competition pull-down experiment with Sulfopin-DTB.

\section{Sulfopin is highly selective}

To evaluate the selectivity of Sulfopin in cells, we assessed its target profile using Covalent Inhibitor Target-site Identification (CITe-Id ${ }^{79}$, Fig. 3A). This chemoproteomic platform enables the identification and quantification of the dose-dependent binding of covalent inhibitors to cysteine residues on a proteome-wide scale. In this competition experiment, live PATU-8988T cells were incubated with Sulfopin $(100,500,1000 \mathrm{nM})$ for $5 \mathrm{~h}$, followed by cell lysis and co-incubation with Sulfopin-DTB $(2 \mu \mathrm{M})$ for $18 \mathrm{~h}$. Following trypsin digestion and avidin enrichment, the DTBmodified peptides were analyzed by multidimensional LC-MS/MS. Out of 162 cysteine residues labeled by Sulfopin-DTB, only Pin1 Cys113 (Fig. 3B; >2 s.d. from the median; Supp. Dataset 2A) exhibited dose-dependent competition (Fig. 3C), indicating the pronounced selectivity of Sulfopin. 

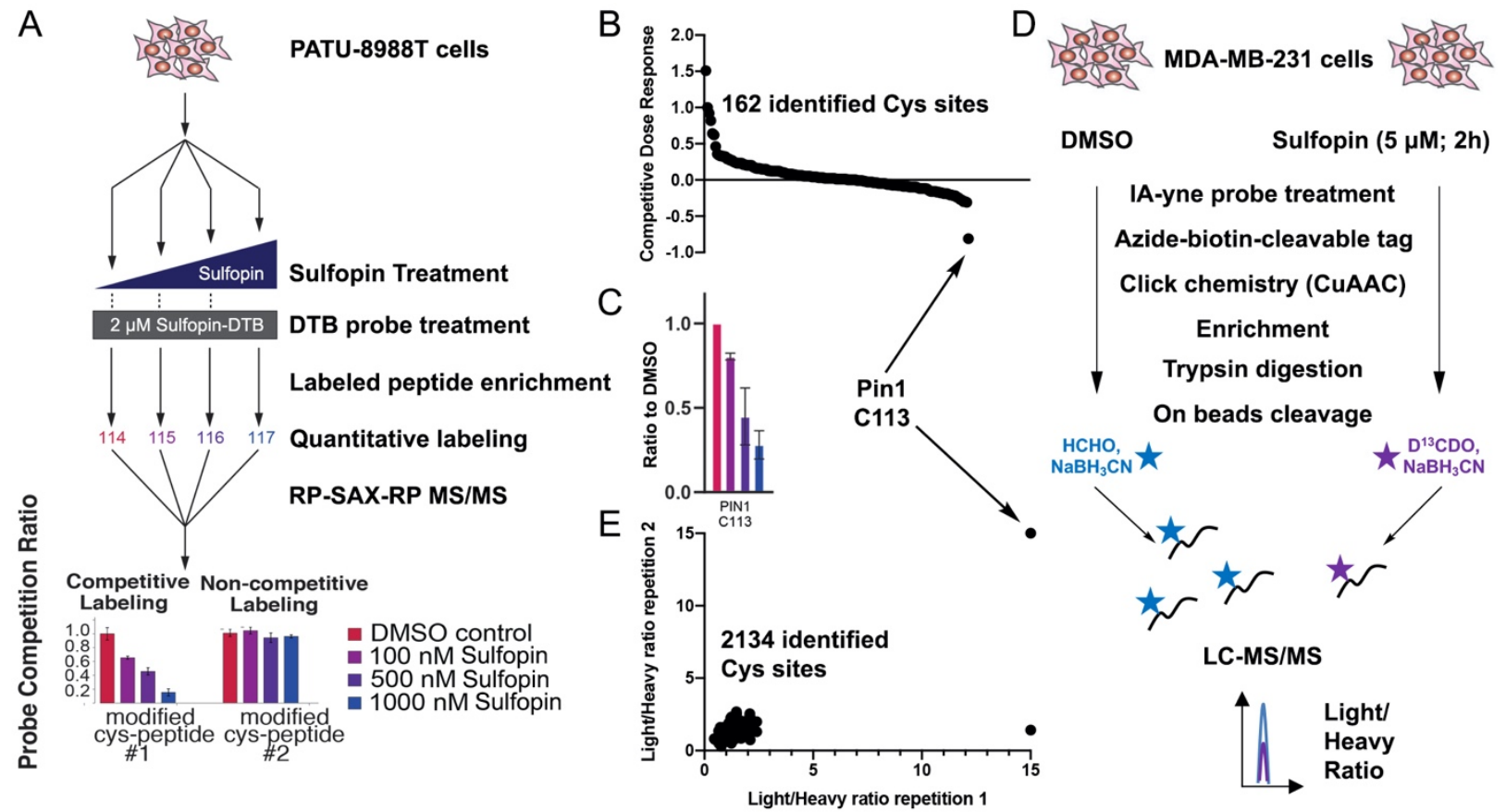

Figure 3. Sulfopin is highly selective for Pin1 in cells.

A. Scheme for Covalent Inhibitor Target-site Identification (CITe-Id) profiling of competitively labeled cysteine residues across the proteome following dose response treatment with Sulfopin. B. CITe-Id results. Out of 162 identified labeled cysteine residues, only C113 in Pin1 is labeled in a dose-dependent manner (see Supp. Dataset 2A for full list of identified peptides). C. Dose dependency of C113 labeling in CITe-ld experiment. D. Scheme for independent rdTOP-ABPP experiment for assessing Sulfopin proteomic selectivity E. Out of 2134 identified cysteines in the experiment, only two cysteines showed light/heavy ratio $>2.5$, of these, one cysteine did not replicate, and only Pin1 C113 showed the maximal ratio of 15 in both replicates.

To further investigate Sulfopin's selectivity, we used a complementary chemoproteomic method. We performed an rdTOP-ABPP experiment to profile its cysteine targets throughout the proteome (Fig. 3D) $)^{80}$. This variant of the isoTOP-ABPP technique enables the site-specific quantification of cysteine binding by label-free covalent inhibitors. In brief, MDA-MB-231 cells were treated with Sulfopin $(5 \mu \mathrm{M}, 2 \mathrm{~h})$, lysed and labeled with a bioorthogonal lodoacetamidealkyne (IA-yne) probe that was then conjugated to a cleavable biotin tag by copper-catalyzed azide-alkyne cycloaddition (CuAAC). After enrichment on beads, the peptides were isotopically derivatized by duplex reductive dimethylation, cleaved and analyzed via LC-MS/MS analysis. We identified Cys113 of Pin1 as the top ranked cysteine that was labeled by Sulfopin at biologically relevant concentration $(5 \mu \mathrm{M})$, with a competition ratio $\mathrm{R}=15$ across two biological replicates (Fig. 3E; Supp. Dataset 2B). All other identified cysteines showed $\mathrm{R}$ values < 2.5. Overall, we used two independent chemoproteomic techniques in two different cell lines to demonstrate that 
Sulfopin has exquisite selectivity for Pin1 Cys113 and is therefore a suitable probe with which to interrogate Pin1's function in cells and in vivo.

Treatment with Sulfopin phenocopies known Pin1 knockout phenotypes

We next assessed whether pharmacological inhibition of Pin1 by Sulfopin could recapitulate two previously reported phenotypes associated with Pin1 genetic knockout. First, Pin1 knockout was reported to abrogate phosphorylation of IRAK1 Thr209 and resensitize radioresistant cancer cells to irradiation ${ }^{31}$. Accordingly, we found that treating radioresistant HeLa cells with Sulfopin significantly resensitized them to irradiation in a dose dependent manner (Fig. 4A), and also decreased IRAK1 Thr209 phosphorylation at concentrations as low as 100nM (Fig. $4 \mathrm{~B}, \mathrm{C})$.

Second, germinal centers (GCs) are sites where B cells proliferate and undergo somatic hypermutation in a BCL6- and Myc-dependent manner. Pin1-deficient mice are reported to display a significant increase in the frequency of GC B cells in response to immunization ${ }^{81}$. To induce GCs and examine Sulfopin's effect on GC B cells, we immunized the hind foot pads of 12 wildtype (WT) mice with ovalbumin coupled to the 4-hydroxy-3-nitrophenylacetyl (NP-OVA). The mice were injected with two doses of Sulfopin (IP; $40 \mathrm{mg} / \mathrm{kg}$ ) or vehicle on days 7 and 9 post immunization, at the peak of the GC response, and on day 11 the mice were sacrificed and frequency of GC B cells in lymph nodes was assessed by flow cytometry (Fig. 4D). In accordance with the previous report ${ }^{81}$, Sulfopin treated mice exhibited 1.34-fold higher proportion of GC B cells compared to mice treated with control vehicle (Fig. 4E). Taken together, these data demonstrate that Sulfopin phenocopies the effects of Pin1 genetic deletion. 
A

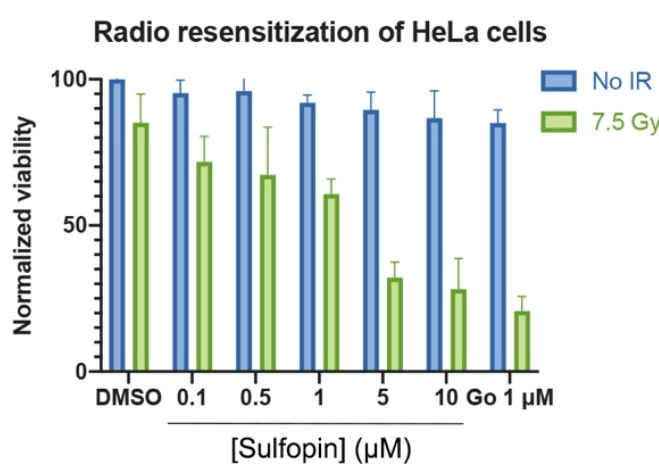

B

$\begin{array}{rlllll}\gamma \mathrm{IR}(\mathrm{Gy}): & - & + & + & + & + \\ \text { Sulfopin }(\mu \mathrm{M}): & - & - & 0.1 & 1.0 & 10\end{array}$

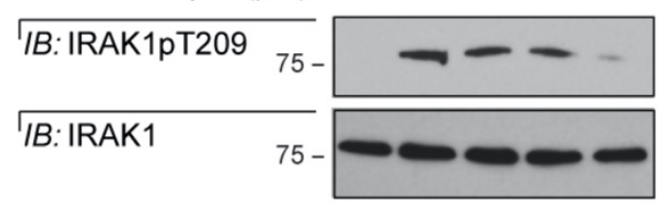

C

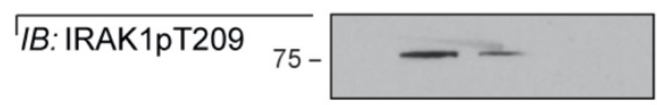

$\mathrm{D}$

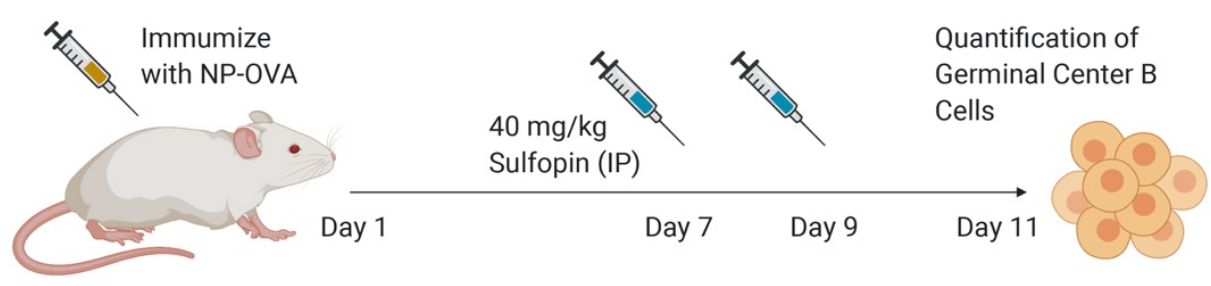

E
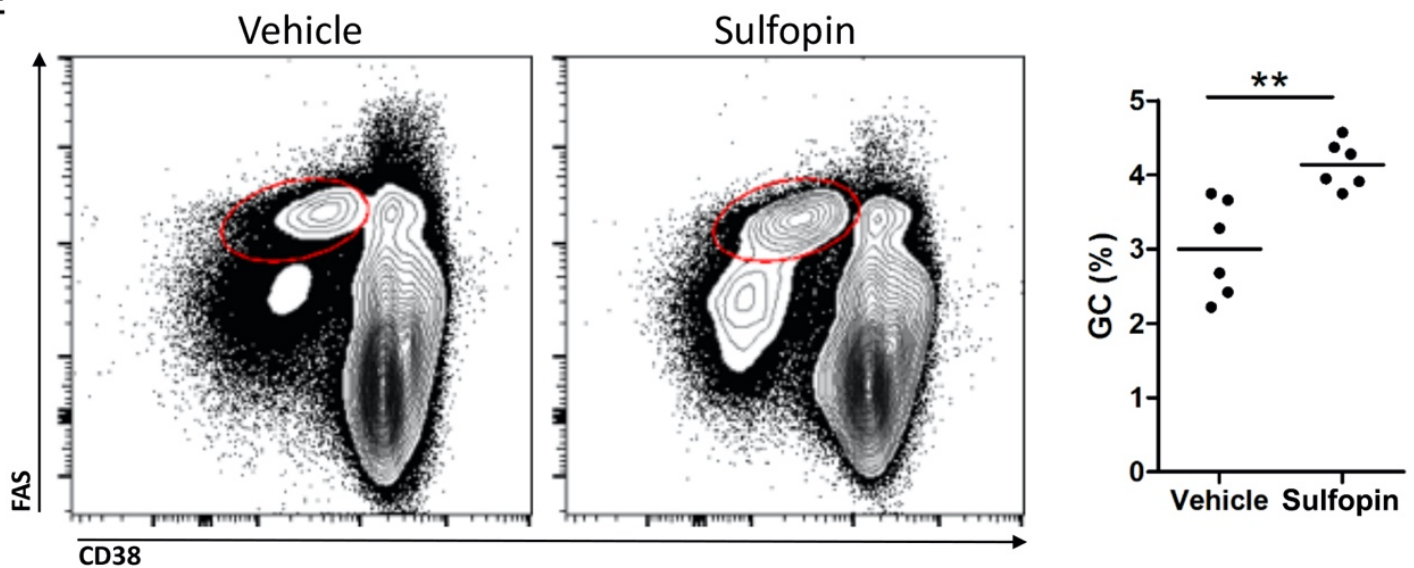

Figure 4. Sulfopin phenocopies Pin1 knockout phenotypes.

A. HeLa cells were treated with either DMSO, Sulfopin, or Go6976 (a Chk1 inhibitor) and exposed to 7.5 Gy IR $1 \mathrm{~h}$ after drug treatment. Viability was assessed 3 days post-IR. Sulfopin shows a dose dependent sensitization of the cells to irradiation. B. Western blot analysis was performed $24 \mathrm{~h}$ post-IR, showing Sulfopin blocked phosphorylation of Thr209 of IRAK1. C. A shorter exposure shows that Sulfopin inhibits IRAK1 phosphorylation already at concentrations of $0.1 \mu \mathrm{M}$. D. A scheme for testing the effect of Sulfopin in vivo on germinal center B cells in response to immunization. E. Representative flow cytometric plots with Vehicle and Sulfopin (left) and quantification (right) of $\mathrm{FAS}^{\mathrm{Hi}} \mathrm{CD} 38^{-}$germinal center (GC) cells in WT mice 11 days after immunization with NP-OVA. ${ }^{* *} p<0.01$, two tailed Student's $t$ test. 
Viability effects of Sulfopin in cancer cell lines

To broadly profile the anti-proliferative activity of Sulfopin, we used the PRISM platform ${ }^{82}$ (Broad Institute) to evaluate its potency against 300 human cancer cell lines. The PRISM method enables high-throughput, pooled screening of mixtures of cell lines, which are each labeled with a 24-nucleotide barcode ${ }^{82}$. In all 300 cancer cell lines profiled, Sulfopin demonstrated limited or no anti-proliferative activity after a 5 -day treatment, with $I_{50}$ values $>3 \mu \mathrm{M}$. This result aligns with our initial cytotoxicity screening, as well as data from the Cancer Dependency Map, in which Pin1 was not identified as a significant genetic dependency in CRISPR-Cas9 and RNAi screens across hundreds of adherent and suspension cancer cell lines (https://depmap.org/portal/). This suggests that the strong single-agent cytotoxicity of previously published Pin1 inhibitors, such as juglone, is likely attributable to off-target effects ${ }^{42,83}$ (Supp Fig. 10).

We next assessed whether Sulfopin treatment might induce antiproliferative activity effects after prolonged treatments (6-8 days). To ensure that target engagement was maintained for the duration of the experiment, we replenished Sulfopin in fresh media every $48 \mathrm{~h}$. We found that Sulfopin treatment impacted the viability of PATU-8988T cells in a Pin1-dependent manner, having no effect on proliferation in the corresponding Pin1 KO cells (Fig. 5A). To evaluate whether this time-dependent growth phenotype was extensible to other cancer types, we performed additional experiments in breast (MDA-MB-468), prostate (PC3), and ovarian (Kuramochi) cancer cell lines. MDA-MB-468 cells showed the most pronounced sensitivity to Sulfopin, which diminished cell viability in a dose- and time-dependent manner, while Sulfopin-AcA did not affect cell proliferation (Fig. 5B). Both PC3 and Kuramochi cells exhibited only very modest sensitivity to Sulfopin treatment.

Given that three-dimensional (3D) culture models can reflect in vivo results better than monolayer cell culture ${ }^{84}$, we next evaluated the anti-proliferative activity of Sulfopin in PATU8988T WT or Pin1 KO cells grown in 3D matrigel domes. Following a 9-day treatment, replenishing compound in media every 3 days, Sulfopin demonstrated modest anti-proliferative activity in PATU-8988T WT Pin1 cells, with no effects observed in PATU-8988T Pin1 KO cells, suggesting an on-target phenotype (Fig. 5C).

Collectively, these data suggest that Pin1 inhibition does not cause proliferation defects at short time points, but that it does moderately affect cell proliferation after prolonged treatments, and in 3D cell culture. 


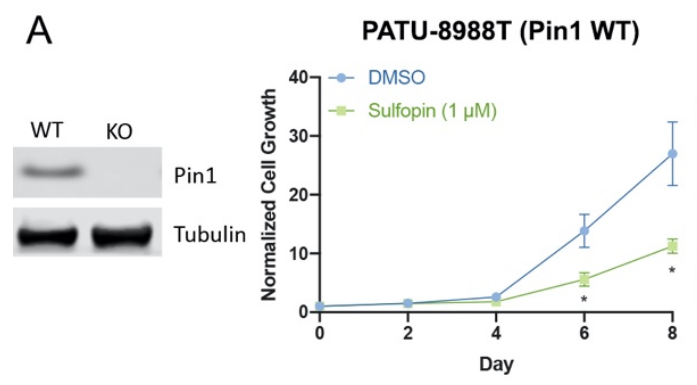

$\mathrm{B}$

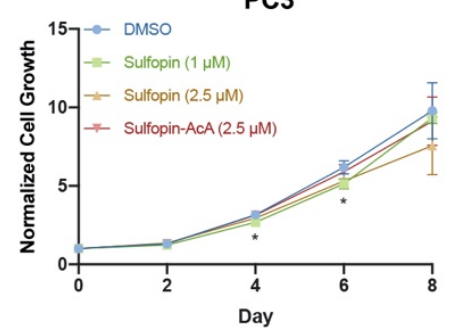

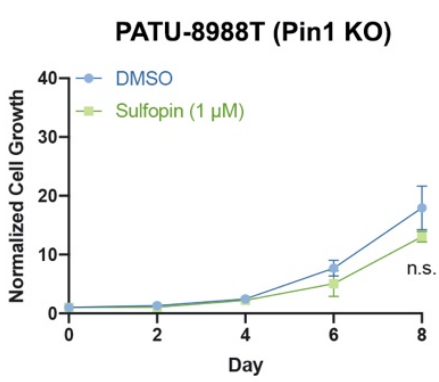

Kuramochi

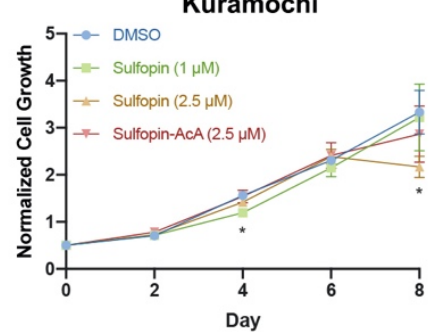

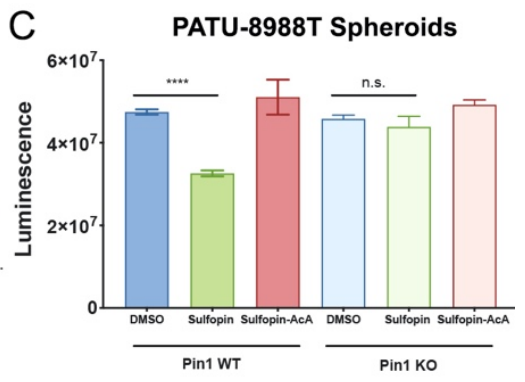

MDA-MB-468

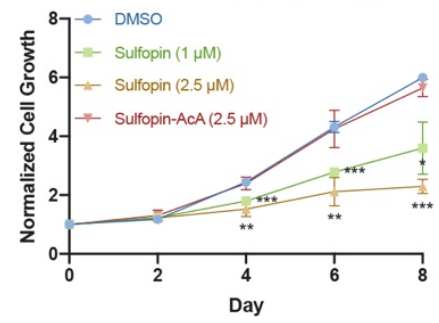

Figure 5. Sulfopin has a Pin1 dependent viability effect on long term exposure.

A. We generated a PATU-8988T Pin1 knockout (KO) cell line, as evidenced by Western blot. Sulfopin (1 $\mu \mathrm{M})$ has a significant effect on cellular viability after 6 and 8 days in the WT PATU-8988T cells, but shows no significant effect on viability in the Pin1 KO cells $(n=3)$. B. Sulfopin shows varying anti-proliferative effects across cancer cell lines, with the most pronounced sensitivity observed in MDA-MB-468 cells ( $n=3$ ). C. Relative viability of PATU-8988T WT and Pin1 KO cells grown in $100 \%$ Matrigel domes following treatment with Sulfopin $(1 \mu \mathrm{M} ; \mathrm{n}=9)$ or Sulfopin-AcA $(1 \mu \mathrm{M} ; \mathrm{n}=9)$. The non-covalent control Sulfopin-AcA shows no effect in any of the tested systems. Statistical significance calculated using Student's t test with unequal variance (n.s. $=p>0.05 ;{ }^{*}=p<0.05 ;{ }^{* *}=p<0.01 ;{ }^{* * *}=p<0.001 ;{ }^{* * * *}=p<0.0001$ ). In all graphs, error bars indicate standard deviation.

\section{Sulfopin downregulates Myc transcriptional activity}

We and others have previously shown that Pin1 regulates the c-Myc oncoprotein ${ }^{85}$, affecting Myc protein stability ${ }^{86}$ as well as its DNA binding and transcriptional activity ${ }^{22,87}$. Pin1 physically interacts with c-Myc ${ }^{86,88}$, altering isomerization of P63 following phosphorylation of S62. We have shown that overexpression of Pin1 can lead to an increase in the transcription of c-Myc target genes while knockdown of Pin1 decreases Myc-dependent transcription ${ }^{22}$.

To test whether Sulfopin affects Myc transcriptional output, we treated Mino B cells with Sulfopin ( $1 \mu \mathrm{M}$; $6 \mathrm{~h}$; in triplicates) or DMSO and performed a global RNA sequencing analysis to detect differentially expressed genes as the result of this perturbation. 206 genes were found to be significantly down-regulated (Fig. 6A; Supp. Dataset 3). We performed a gene set enrichment analysis of these genes using Enrichr ${ }^{89}$, against a dataset of genes identified by ChIP-seq (chromatin immunoprecipitation followed by sequencing) for various transcription factors. Myc target genes in K562 cells and HeLa-S3 cells appeared as the most enriched set and the third 
most enriched set, respectively (adjusted p-value of $1.99 \times 10^{-16}$ and $2.00 \times 10^{-13}$ respectively; Fig. $6 \mathrm{~B})$, validating a significant downregulation of Myc's transcriptional signature by Sulfopin. To further validate the effect of Sulfopin on Myc transcriptional activity, we co-transfected HEK-293 cells with a Myc reporter construct (4x-Ebox-Luciferase) and Pin1. As expected, Pin1 expression increased Myc transcriptional activity, while treatment with $2 \mu \mathrm{M}$ Sulfopin for $48 \mathrm{~h}$ resulted in a significant reduction in relative luciferase activity as compared to DMSO control (Fig. 6C). Together these results suggest that treatment with Sulfopin downregulates Myc target genes and, thus, points to Myc-driven cancers as natural candidates for its therapeutic application. Accordingly, we next evaluated Sulfopin in in vivo models of MYCN-driven neuroblastoma (NB).

A

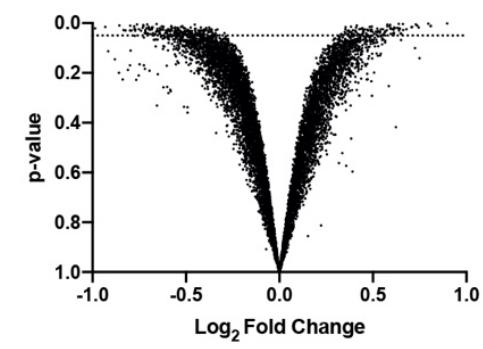

B

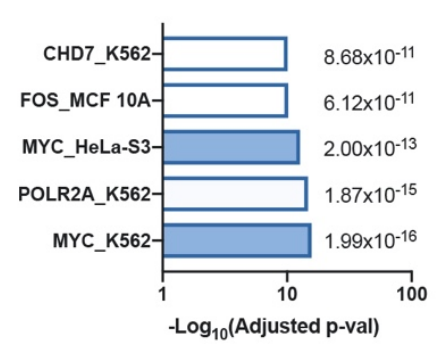

C

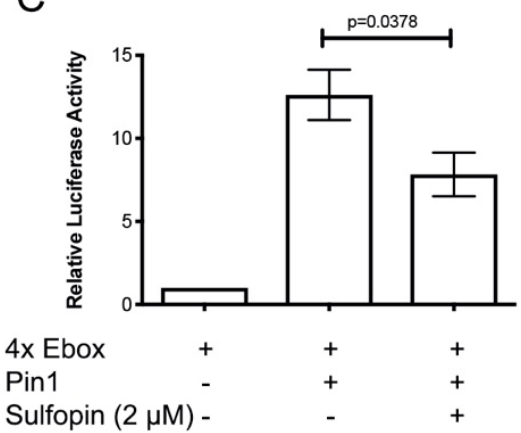

Figure 6. Sulfopin downregulates Myc transcription.

A. Results of an RNA-sequencing experiment, comparing the change in RNA levels between Mino B cells treated with either Sulfopin ( $1 \mu \mathrm{M}, 6 \mathrm{~h}$, in triplicates) or DMSO. Each dot presents the $\log _{2}$ fold change of a transcript ( $x$-axis) vs. the $p$-value for significance of that change (Student's $t$ test; $y$-axis). The dotted line indicates $p=0.05$. 206 genes were significantly down regulated. B. Results of a gene set enrichment analysis using Enrichr against the ENCODE TF ChIP-seq set. Two of the most enriched sets are Myc target genes from different cell lines. C. HEK293 cells were transfected with a 4x Ebox-luciferase reporter for Myc transcriptional activity levels. Co-transfection with Pin1 increased reporter activity, while $48 \mathrm{~h}$ treatment with Sulfopin significantly (Student's t test) reduced this activity compared to DMSO.

Sulfopin blocks tumor initiation and progression in MYCN-driven zebrafish models of neuroblastoma

To evaluate Sulfopin's effects on Myc-driven cancers, we turned to a zebrafish model of neuroblastoma ${ }^{90-92}$, a pediatric malignancy derived from the peripheral sympathetic nervous system (PSNS) ${ }^{93}$. During the development of normal zebrafish embryos, neural crest-derived PSNS neuroblasts form the primordial superior cervical ganglia (SCG) and intrarenal gland (IRG) at the age of 3 to 7 days post fertilization (dpf), can be visualized using the $d \beta h$ :EGFP fluorescent reporter ${ }^{91}$ (Fig. 7A). Overexpression of the $\mathrm{MYCN}$ oncogene, which is the oncogenic driver in 
approximately $20 \%$ of human high-risk neuroblastomas, causes the fish to develop neuroblast hyperplasia in the PSNS of Tg(dßh:MYCN;dßh:EGFP) transgenic zebrafish (Fig. 7A upper right). These neuroblast hyperplasia rapidly progress into fully transformed tumors that faithfully resemble human high-risk neuroblastoma ${ }^{90-92}$. When Sulfopin was added to the fish water containing $\mathrm{Tg}(\mathrm{d} \beta \mathrm{h}: \mathrm{MYCN} ; \mathrm{d} \beta \mathrm{h}:$ EGFP) zebrafish (at concentrations of $25-100 \mu \mathrm{M}$ ), the neuroblastoma-initiating hyperplasia was significantly suppressed and fully transformed neuroblastoma did not develop over the treatment period (Fig. 7A,B). This indicates that Sulfopin blocks neuroblastoma initiation in vivo in this tumor model. In addition, no evidence of toxicity was observed in embryos treated with Sulfopin at these concentrations, further supporting our findings in mice that Sulfopin is well tolerated by healthy tissues in vivo.

We then assessed the anti-tumor activity of Sulfopin on the maintenance of fully transformed neuroblastoma cells in vivo in primary tumor derived allograft (PDA) models constructed in transplanted zebrafish embryos. EGFP-labeled neuroblastoma cells were dissected from four-month-old $\mathrm{Tg}(\mathrm{d} \beta \mathrm{h}: \mathrm{MYCN}$; $\mathrm{d} \beta \mathrm{h}: \mathrm{EGFP})$ donor zebrafish, disaggregated, counted and 200-400 GFP-labeled tumor cells were injected intravenously into the Duct of Cuvier (common cardinal vein) of $2 \mathrm{dpf}$ zebrafish embryos ${ }^{94}$. One day after injection, $100 \mu \mathrm{M}$ Sulfopin or the DMSO control was added to the fish water containing embryos bearing the transplanted EGFP-labeled neuroblastoma cells. After five days, we quantified the area of the EGFP-labeled tumor mass in treated embryos, and discovered that tumor masses in the DMSO-treated embryos grew larger over the five days of treatment, while the tumor masses decreased in size in the Sulfopin treated embryos (Fig. 7C,D). Hence, Sulfopin treatment not only suppressed MYCNdriven neuroblastoma initiation, but also suppressed the growth and survival in vivo of transplants of fully transformed primary neuroblastoma tumor cells.

\section{Sulfopin blocks tumor initiation and progression in a MYCN-mouse model of neuroblastoma}

Following the encouraging results in the zebrafish models, we also assessed the effects of Sulfopin in a murine model of neuroblastoma - the Th-MYCN GEM (genetically engineered mouse) model of neuroblastoma, in which human MYCN is expressed under the tyrosine hydroxylase promoter ${ }^{95}$. The Th-MYCN faithfully recapitulate the major molecular and histopathologic features of high-risk MYCN-amplified neuroblastoma, and the model has been widely used for therapeutics studies. The study was performed using both male and female hemizygous mice, which spontaneously developed palpable tumors at 50 to 130 days with a $25 \%$ penetrance. Once tumors were palpable, mice were randomly assigned to treatment groups, and 
treated once per day with either vehicle or $40 \mathrm{mg} / \mathrm{kg}$ Sulfopin. In this aggressive model, we assessed the overall survival of the mice. Treated mice showed significant $(p=0.0127)$ increase in overall survival, with an average increase of 10 days for Sulfopin treated mice (Fig. 7E).

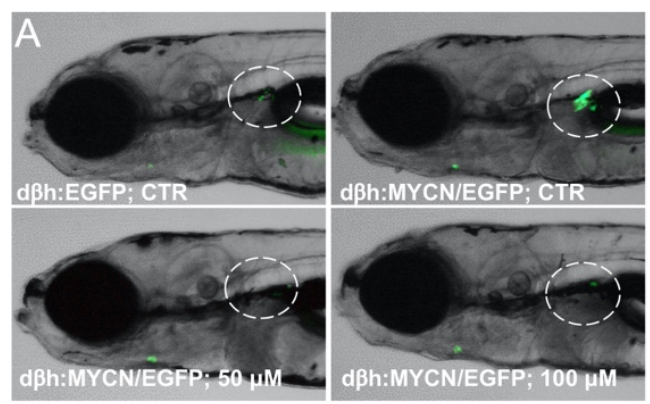

B

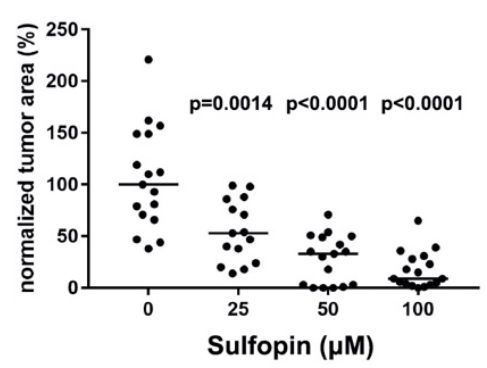

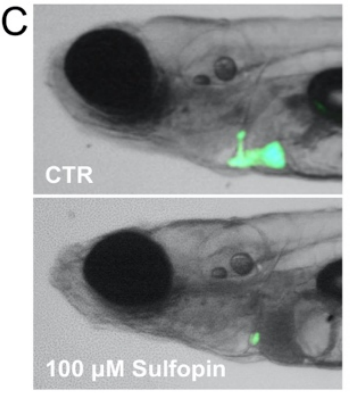

$\mathrm{D}$

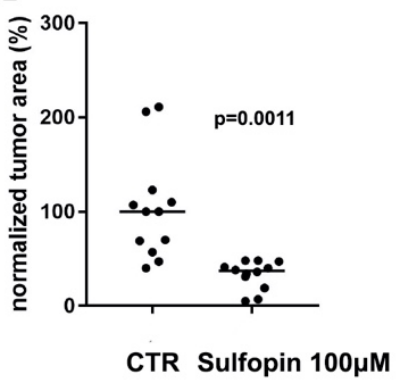

$E$

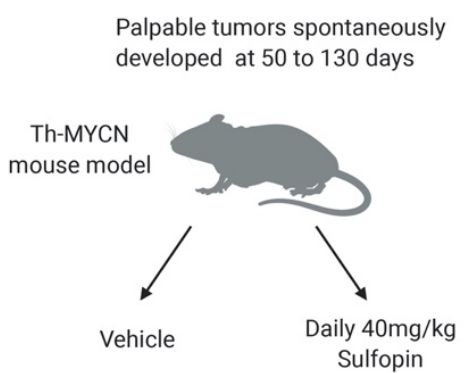

Survival trial (Th-MYCN model)

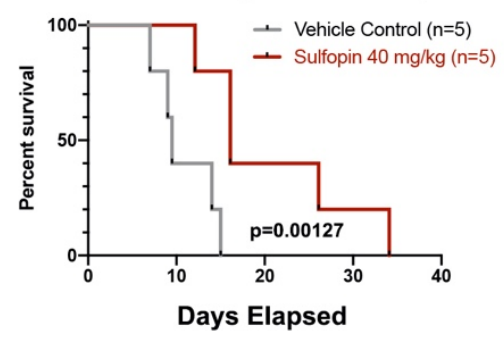

Figure 7. Sulfopin abrogate MYCN driven neuroblastoma growth in vivo. A. PSNS cells in the primordial superior cervical ganglia (SCG) and intrarenal gland (IRG; highlighted by dotted circles) in representative embryos of $\mathrm{Tg}(\mathrm{d} \beta \mathrm{h}: \mathrm{EGFP})$ (upper left) and $\mathrm{Tg}(\mathrm{d} \beta \mathrm{h}: \mathrm{MYCN} ; \mathrm{d} \beta \mathrm{h}: \mathrm{EGFP}$ ) (upper right) transgenic zebrafish at $7 \mathrm{dpf}$. Representative $7 \mathrm{dpf} \mathrm{Tg}(\mathrm{d} \beta \mathrm{h}: \mathrm{MYCN}$; $\mathrm{d} \beta \mathrm{h}$ :EGFP) zebrafish treated with $50 \mu \mathrm{M}$ Sulfopin (bottom left) and $100 \mu \mathrm{M}$ Sulfopin (bottom right). The primordial SCG plus IRG areas are highlighted by dotted circles. B. Quantification of GFP+ cells in the primordial SCG and IRG of $7 \mathrm{dpf}$ $\mathrm{Tg}(\mathrm{d} \beta \mathrm{h}: \mathrm{MYCN} ; \mathrm{d} \beta \mathrm{h}: \mathrm{EGFP})$ embryos treated with Sulfopin at multiple doses. A Mann-Whitney test with confidence intervals of $95 \%$ was used for the analysis of significance ( $p$-value) and the quantitative data are reported as median. C. Representative zebrafish embryos transplanted with neuroblastoma cells isolated from four-month old $\mathrm{Tg}(\mathrm{d} \beta \mathrm{h}: \mathrm{MYCN} ; \mathrm{d} \beta \mathrm{h}: \mathrm{EGFP})$ donor zebrafish and treated with DMSO control (upper) or $100 \mu \mathrm{M}$ Sulfopin added to the fish water (lower). D. Quantification of EGFP-positive tumor area in zebrafish embryos treated with DMSO control and $100 \mu \mathrm{M}$ Sulfopin added to the fish water. A MannWhitney test with confidence intervals of $95 \%$ was used for the analysis of significance ( $p$-value) and the quantitative data are reported as median. E. Survival trials in Th- $M Y C N$ mice. Mice were randomly assigned to treatment groups, and treated daily with either vehicle or $40 \mathrm{mg} / \mathrm{kg}$ Sulfopin, as soon as the tumors were palpable. Treated mice showed significant $(p=0.0127)$ increase in overall survival, with an average increase of 10 days survival. 


\section{Discussion}

Despite extensive, decades-long efforts to discover Pin1 inhibitors, no approach has yielded a compound capable of selectively blocking Pin1 in vivo. Here, we describe the development and in vivo characterization of Sulfopin, a highly selective and potent, covalent Pin1 inhibitor with low inherent reactivity and negligible toxicity, which blocks tumor initiation and progression in both zebrafish and mouse models of neuroblastoma.

While inhibitors such as juglone, ATRA, ATO and KPT-6566 pioneered the investigation of Pin1 in cancer-related contexts, they all exert their anti-cancer effects in part through Pin1independent mechanisms ${ }^{41}$ that include DNA-damage ${ }^{39}$, induction of Pin1 degradation ${ }^{37}$ or by directly blocking transcription ${ }^{83}$. Our recently reported covalent peptide inhibitor BJP-06-005-3 ${ }^{43}$, while selective in cells, is not suitable for in vivo application. In contrast, Sulfopin is highly specific for Pin1, as we established here using multiple orthogonal experimental strategies. Therefore, this highly selective in vivo tool allowed us to investigate Pin1 as a bona fide drug target in cancer for the first time. Thus far, Pin1 has proven a challenging target for both ligand- ${ }^{74}$ and structurebased ${ }^{76-78}$ approaches, even in studies employing high-throughput-screenings of up to one million compounds ${ }^{76}$. This is in part due to the shallow nature of the Pin 1 active site, which is evolved to bind peptides, further complicated by the phosphate binding site, which favors negatively charged moieties. To overcome this hurdle, we screened a library of electrophilic fragments, which can compensate for sparse protein interfaces by irreversibly binding to the Pin1 Cys113. The screen resulted in a relatively high hit rate of $11 \%$ (111 compounds with $>50 \%$ labeling) compared to previous screens ${ }^{62}$. This might be explained by the high reactivity of Cys113, which was found amongst the most reactive cysteines in several chemoproteomic campaigns ${ }^{67}$.

A major finding during our hit optimization campaign was that intrinsic warhead reactivity of Pin1 binders did not correlate with potency (Supp. Fig. 5). Despite the high structural similarity of the optimized compounds, their reactivities spanned over 30-fold (Supp. Table 2), with Sulfopin having the lowest reactivity of the best binders (Fig. 1C), with reactivity nearing that of acrylamides (Supp. Fig. 11). This low reactivity manifested in exquisite cellular selectivity. Two separate chemoproteomic approaches, performed in two different cell lines, both identified Pin1 as the sole target of Sulfopin by a wide margin (Fig. 3). This result is rare for covalent inhibitors ${ }^{79}$ and even for FDA approved covalent drugs ${ }^{96}$.

This proteomic selectivity for Pin1 allowed us to show that short-term Pin1 inhibition does not induce cytotoxicity, despite previous results reported with less selective inhibitors ${ }^{39,97-102}$. 
Juglone, for instance, has identical LD $_{50}$ for cell lines lacking Pin1 ${ }^{42,43}$ (Supp. Fig. 10). Sulfopin induced little to no cytotoxicity across a panel of 300 cancer cell lines (PRISM screen), though it significantly impacted cancer cell growth after prolonged treatments (6-8 days; Fig. 5A,B) and in 3D-cell culture (Fig. 5C). The irreversible binding of Sulfopin allowed us to demonstrate target engagement in cells and in vivo (Fig. 2), showing that its favorable PK/PD profile (Supp. Table 5) translated to complete in vivo target engagement and target modulation as demonstrated by the ability of Sulfopin to phenocopy previous genetic results in vivo (Fig. 4). This result underscores the utility of Sulfopin as an in vivo tool compound, with broad applicability to domains such as immunology, and as we later show, cancer biology.

In agreement with previous studies ${ }^{22,86}$, our RNA sequencing experiment suggested Myc target gene downregulation as a major consequence of Pin1 inhibition (Fig. 6A,B). This conclusion is supported by transcriptional suppression in the Myc luciferase reporter assay (Fig. 6C), and the demonstrated efficacy of Pin1 inhibition in various MYCN-driven cancer models (Fig. 7). However, given that Pin1 plays a central role in numerous signaling pathways, it is likely that Mycdownregulation is not the only mechanism at play. Indeed, additional transcription factors showed significant downregulation in the RNA-seq dataset, including RelA (Supp. Dataset 4) which was also previously linked to Pin ${ }^{9,103}$ and could contribute to oncogenesis. Other pathways that were previously linked to Pin1 have also been implicated in neuroblastoma 104,105. RNA-seq experiments in additional cell lines (and perhaps additional time points) might shed more light on other processes regulated by Pin1.

Further investigation is needed to establish whether the therapeutic efficacy of Sulfopin can be further enhanced. For instance, we have yet to reach dose limiting toxicity with Sulfopin, and observed dose-dependent responses in zebrafish (Fig. 7B), indicating that treatment with higher doses might enable more pronounced effects in mice. Furthermore, its favorable toxicity profile could enable the use of Sulfopin in combination with other drugs. Since Pin1 regulates numerous cellular pathways, it stands to reason that Pin1 inhibition may be synthetic lethal with the loss of other targets. Ongoing studies will clarify the full therapeutic potential of Sulfopin.

In summary, we present a potent and selective, covalent Pin1 inhibitor with in vivo activity and no toxicity that allows investigation of Pin1 biology in physiologically relevant models in health and disease. Using Sulfopin as a pharmacological tool will enable the study of the many diverse processes that are regulated by Pin1. 


\section{Methods}

\section{Electrophile library screening}

993 compounds as 20 mM DMSO stocks in 384 well-plate format were transferred into a 384 well plate working copy by combining $0.5 \mu \mathrm{L}$ of five compounds per well. The catalytic domain of Pin 1 $(2 \mu \mathrm{M})$ in $20 \mathrm{mM}$ Tris, $75 \mathrm{mM} \mathrm{NaCl}, \mathrm{pH} 7.5$ was incubated with $200 \mu \mathrm{M}$ for each compound and moderately shaken for $24 \mathrm{~h}$ at $4^{\circ} \mathrm{C}$. The reaction was stopped by the addition of formic acid to $0.4 \%$ final concentration. The LC/MS runs were performed on Waters ACUITY UPLC class $\mathrm{H}$, in positive ion mode using electrospray ionization. UPLC separation using C4 column (300 $\AA, 1.7$ $\mu \mathrm{M}, 21 \mathrm{~mm} \times 100 \mathrm{~mm}$ ). the column was held at $40^{\circ} \mathrm{C}$, and the autosampler at $10^{\circ} \mathrm{C}$. Mobile solution A was $0.1 \%$ formic acid in water and mobile phase $B$ was $0.1 \%$ formic acid in acetonitrile. Run flow was $0.4 \mathrm{~mL} / \mathrm{min}$. Gradient used for BSA was $20 \%$ B for 2 minutes increasing linearly to $60 \%$ $\mathrm{B}$ for 4 minutes holding at $60 \% \mathrm{~B}$ for 2 minutes, changing to $0 \% \mathrm{~B}$ in 0.1 minutes and holding at $0 \%$ for 1.9 minutes. Gradient for the other proteins was $20 \%$ B for 2 minutes increasing linearly to $60 \% \mathrm{~B}$ for 3 minutes holding at $60 \% \mathrm{~B}$ for 1.5 minutes, changing to $0 \% \mathrm{~B}$ in 0.1 minutes and holding at $0 \%$ for 1.4 minutes. The mass data was collected at a range of $600-1300 \mathrm{~m} / \mathrm{z}$. Desolvation temperature was $500^{\circ} \mathrm{C}$ with a flow rate of 1000 liter/hour. The voltage used were $0.69 \mathrm{kV}$ for the capillary and $46 \mathrm{~V}$ for the cone. Raw data was processed using openLYNX and deconvoluted using MaxEnt. Labeling assignment was performed as previously described ${ }^{62}$.

\section{Covalent Docking}

Covalent docking was performed using DOCKovalent $3.7^{73}$ against 16 structures of Pin1. PDB codes: 1PIN, 2ITK, 2Q5A, 2XP3, 2ZQV, 2ZR4, 3IK8, 3KAB, 3KCE, 3NTP, 3ODK, 3OOB, 3TC5, 3TCZ, 3TDB, 3WH0. The docked compounds include seven sulpholane hits from the electrophilic library with the following IDs: PCM-0102138, PCM-0102178, PCM-0102105, PCM-0102832, PCM-0102313, PCM-0102760, PCM-0102755. The covalent bond length was set to $1.8 \AA$ and the two newly formed bond angles to $\mathrm{C} \beta-\mathrm{S}_{Y}-\mathrm{C}=109.5 \pm 5^{\circ}$ and $\mathrm{S} Y$-C-Ligatom $=109.5 \pm 5^{\circ}$.

\section{Thiol reactivity assay}

$50 \mu \mathrm{M}$ DTNB was incubated with $200 \mu \mathrm{M}$ TCEP in $20 \mathrm{mM}$ sodium phosphate buffer $\mathrm{pH}$ 7.4, 150 $\mathrm{mM} \mathrm{NaCl}$, for 5 minutes at room temperature, in order to obtain $\mathrm{TNB}^{2-} .200 \mu \mathrm{M}$ compounds were subsequently added to the $\mathrm{TNB}^{2-}$, followed by immediate UV absorbance measurement at 412 $\mathrm{nm}$ at $37^{\circ} \mathrm{C}$. The UV absorbances were acquired every 15 minutes for $7 \mathrm{~h}$. The assay was performed in a 384 well-plate using a Tecan Spark10M plate reader. Background absorbance of 
compounds was subtracted by measuring the absorbance at $412 \mathrm{~nm}$ of each compound in the same conditions without DTNB. Compounds were measured in triplicates. The data was fitted to a second order reaction equation such that the rate constant $k$ is the slope of $\ln ([A][B 0] /[B][A 0])$. Where $[\mathrm{A} 0]$ and $[\mathrm{B} 0]$ are the initial concentrations of the compound $(200 \mu \mathrm{M})$ and $\mathrm{TNB}^{2-}(100 \mu \mathrm{M})$ respectively, and $[A]$ and $[B]$ are the remaining concentrations as a function of time as deduced from the spectrometric measurement. Linear regression using Prism was performed to fit the rate against the first $4 \mathrm{~h}$ of measurements.

\section{Pin1 expression and purification}

A construct of full-length human Pin1 in a pET28 vector was overexpressed in E. coli BL21 (DE3) in LB medium in the presence of $50 \mathrm{mg} / \mathrm{ml}$ of kanamycin. Cells were grown at $37^{\circ} \mathrm{C}$ to an OD of 0.8 , cooled to $17^{\circ} \mathrm{C}$, induced with $500 \mu \mathrm{M}$ isopropyl-1-thio-D-galactopyranoside, incubated overnight at $17^{\circ} \mathrm{C}$, collected by centrifugation, and stored at $-80^{\circ} \mathrm{C}$. Cell pellets were sonicated in buffer $\mathrm{A}$ (50 mM hepes 7.5, $500 \mathrm{mM} \mathrm{NaCl}, 10 \%$ glycerol, $20 \mathrm{mM}$ Imidazole, and $7 \mathrm{mM} \mathrm{BME}$ ) and the resulting lysate was centrifuged at 30,000 xg for $40 \mathrm{~min}$. Ni-NTA beads (Qiagen) were mixed with lysate supernatant for $30 \mathrm{~min}$ and washed with buffer $\mathrm{A}$. Beads were transferred to an FPLCcompatible column and the bound protein was washed with $15 \%$ buffer $B$ (50 mM hepes 7.5, 500 $\mathrm{mM} \mathrm{NaCl}, 10 \%$ glycerol, $250 \mathrm{mM}$ Imidazole, and $3 \mathrm{mM} \mathrm{BME}$ ) and eluted with $100 \%$ buffer $\mathrm{B}$. Thrombin was added to the eluted protein and incubated at $4^{\circ} \mathrm{C}$ overnight. The sample was concentrated and passed through a Superdex 200 10/300 column (GE helathcare) in a buffer containing $20 \mathrm{mM}$ HEPES, $\mathrm{pH} 7.5,150 \mathrm{mM} \mathrm{NaCl}, 5 \%$ glycerol, and $1 \mathrm{mM}$ TCEP. Fractions were pooled, concentrated to approximately $37 \mathrm{mg} / \mathrm{ml}$ and frozen at $-80^{\circ} \mathrm{C}$.

\section{Pin1 crystallization and soaking}

Apo protein at a final concentration of $1 \mathrm{mM}$ was crystallized by sitting-drop $(200 \mathrm{~nL}+200 \mathrm{~nL})$ vapor diffusion at $20^{\circ} \mathrm{C}$ in the following crystallization buffer: $3 \mathrm{M} \mathrm{NH}_{4} \mathrm{SO}_{4}, 100 \mathrm{mM} \mathrm{HEPES}-\mathrm{pH} 7.5$, $150 \mathrm{mM} \mathrm{NaCl}, 1 \%$ PEG400, and $10 \mathrm{mM}$ DTT. A volume of $200 \mathrm{~nL}$ of $1 \mathrm{mM}$ Sulfopin was added directly to crystals for soaking at $20^{\circ} \mathrm{C}$ for $16 \mathrm{~h}$. Crystals were transferred briefly into crystallization buffer containing $25 \%$ glycerol prior to flash-freezing in liquid nitrogen.

\section{Crystallization data collection and structure determination}

Diffraction data from complex crystals were collected at beamline 24ID-C of the NE-CAT at the Advanced Photon Source at the Argonne National Laboratory. Data sets were integrated and scaled using $\mathrm{XDS}^{106}$. Structures were solved by molecular replacement using the program 
Phaser ${ }^{107}$ and the search model PDB entry 1PIN. Iterative manual model building and refinement using Phenix ${ }^{108}$ and Coot $^{109}$ led to the final models (Supp. Table 4).

Fluorescence polarization (FP) assay

Binding affinity to Pin1 was determined using a fluorescence polarization (FP) assay to assess competition with an N-terminal fluorescein-labeled peptide (peptide core structure: Bth-D-pThrPip-Nal), which was synthesized by Proteintech. The indicated concentrations of candidate compounds were pre-incubated for $12 \mathrm{~h}$ at $4^{\circ} \mathrm{C}$ with a solution containing $250 \mathrm{nM}$ glutathione Stransferase (GST)-Pin1, $5 \mathrm{nM}$ of fluorescein-labeled peptide probe, $10 \mu \mathrm{g} / \mathrm{ml}$ bovine serum albumin, $0.01 \%$ Tween-20 and $1 \mathrm{mM}$ DTT in a buffer of $10 \mathrm{mM}$ HEPES, $10 \mathrm{mM} \mathrm{NaCl}$ and $1 \%$ glycerol (pH 7.4). Measurements of FP were made in black 384-well plates (Corning) using an EnVision reader. $\mathrm{K}_{\mathrm{i}}$ values obtained from the FP assay results were derived from the Kenakin $\mathrm{K}_{\mathrm{i}}$ equation: Kenakin $K_{i}=(L b)\left(E C_{50}\right)\left(K_{d}\right) /(L o)(R o)+L b\left(R o-L o+L b-K_{d}\right)$, where $K_{d}[M]: K_{d}$ of the probe, $\mathrm{EC}_{50}[\mathrm{M}]$ : concentration of unlabeled compound that results in $50 \%$ inhibition of binding (obtained from FP assay), total tracer Lo [M]: probe concentration in FP, bound tracer Lb [M]: $85 \%$, fraction of probe bound to Pin1, total receptor Ro [M]: Pin1 concentration in the FP assay, as described ${ }^{37,110}$.

\section{Pin1 PPlase activity assay}

Inhibition of Pin1 isomerase activity was determined using the chymotrypsin-coupled PPlase assay, using GST-Pin1 and Suc-Ala-pSer-Pro-Phe-pNA peptide substrate, as described previously ${ }^{75}$. GST-Pin1 was pre-incubated with the indicated concentrations of compound for 12 $\mathrm{h}$ at $4^{\circ} \mathrm{C}$ in buffer containing $35 \mathrm{mM}$ HEPES ( $\left.\mathrm{pH} 7.8\right), 0.2 \mathrm{mM} \mathrm{DTT}$, and $0.1 \mathrm{mg} / \mathrm{mL}$ BSA. Immediately before the assay was started, chymotrypsin (final concentration of $6 \mathrm{mg} / \mathrm{mL}$ ) was added, followed by the addition of the peptide substrate (Suc-Ala-pSer-Pro-Phe-pNA peptide substrate, final concentration $50 \mathrm{mM}$ ). The $K_{\mathrm{i}}$ value obtained from the PPlase assay was derived from the Cheng-Prusoff equation, $K_{i}=I C_{50} /\left(1+S / K_{m}\right)$, where $K_{m}$ is the Michaelis constant for the peptide substrate, $S$ is the initial concentration of the substrate in the assay, and $\mathrm{IC}_{50}$ is the halfminimal inhibitory concentration of the inhibitor.

\section{Cell Culture and Reagents.}

PATU-8988T (DSMZ), MDA-MB-468 (ATCC), and HeLa (ATCC) cells were cultured in Dulbecco's modified Eagle's medium (DMEM) supplemented with 10\% fetal bovine serum (FBS, Sigma) and 1\% penicillin/streptomycin. PC3 (ATCC), IMR32 (ATCC) and Kuramochi (Panagiotis A. 
Konstantinopoulos's laboratory) cells were cultured in RPMI 1640 medium with L-glutamine, supplemented with $10 \% \mathrm{FBS}$ and $1 \%$ penicillin/streptomycin. All cell lines were cultured at $37^{\circ} \mathrm{C}$ in a humidified chamber in the presence of $5 \% \mathrm{CO}_{2}$. All cell lines were tested for the absence of Mycoplasma infection on a monthly basis.

\section{Immunoblotting.}

Whole cell lysates for immunoblotting were prepared by pelleting cells from each cell line at $4^{\circ} \mathrm{C}$ $(300 \mathrm{~g})$ for 5 minutes. The resulting cell pellets were washed $1 \mathrm{x}$ with $5 \mathrm{~mL}$ ice-cold PBS and then resuspended in the indicated cell lysis buffer. Lysates were clarified at 14,000 rpm for 15 minutes at $4^{\circ} \mathrm{C}$ prior to quantification by BCA assay (Pierce, cat\#23225). Whole cell lysates were loaded into Bolt 4-12\% Bis-Tris Gels (Thermo Fisher, cat\#NW04120BOX) and separated by electrophoreses at $95 \mathrm{~V}$ for $1.5 \mathrm{~h}$. The gels were transferred to a nitrocellulose membrane using the iBlot Gel Transfer at P3 for 6 minutes (Thermo Fisher, cat\#IB23001) and then blocked for $1 \mathrm{~h}$ at room temperature in Odyssey blocking buffer (LICOR Biosciences, cat\#927-50010). Membranes were probed using antibodies against the relevant proteins at $4^{\circ} \mathrm{C}$ overnight in $20 \%$ Odyssey Blocking Buffer in 1x TBST. Membranes were then washed three times with 1x TBST (at least 5 minutes per wash) followed by incubation with the IRDye goat anti-mouse (LICOR, cat\#926-32210) or goat anti-rabbit (LICOR, cat \#926-32211) secondary antibody (diluted $1: 10,000$ ) in $20 \%$ Odyssey Blocking Buffer/1x TBST for $1 \mathrm{~h}$ at room temperature. After three washes with 1x TBST (at least 5 minutes per wash), the immunoblots were visualized using the ODYSSEY Infrared Imaging System (LICOR). Antibodies used against various proteins were as follows: Pin1 (1:1,000, Cell Signaling cat\#3722), $\alpha$-Tubulin (1:1,000, Cell Signaling cat\#3873), IRAK1 (Cell Signaling Technology \#4504), IRAK1 pT209 (Assay Biotech \#A1074).

\section{Lysate Pull-Down with Sulfopin-DTB.}

The indicated cells were lysed in 50 mM Hepes, pH 7.4, 1 mM EDTA, 10\% glycerol, 1 mM TCEP, $150 \mathrm{mM} \mathrm{NaCl}, 1 \mathrm{mM}$ EDTA, 0.5\% NP-40, and protease inhibitor tablet (Roche cat\#4693159001). After clarifying (14,000 rpm for $15 \mathrm{~min})$, lysates were incubated with the indicated concentrations of Sulfopin-DTB for $1 \mathrm{~h}$ at $4^{\circ} \mathrm{C}$, using $500 \mu \mathrm{g}$ of lysate per sample. Lysates were then incubated with streptavidin agarose resin (30 $\mu \mathrm{L}$ of 1:1 beads:lysis buffer slurry) (Thermo scientific, cat\#20349) for $1.5 \mathrm{~h}$ at $4^{\circ} \mathrm{C}$. Beads were washed four times with $500 \mu \mathrm{L}$ of buffer $(50 \mathrm{mM}$ Hepes, $\mathrm{pH} 7.5,10 \mathrm{mM} \mathrm{NaCl}, 1 \mathrm{mM}$ EDTA, $10 \%$ glycerol), then pelleted by centrifugation and dried. The beads were boiled for 5 minutes at $95^{\circ} \mathrm{C}$ in $30 \mu \mathrm{L}$ of $2 x \mathrm{LDS}+5 \% \beta$-mercaptoethanol. Lysates were probed for specified proteins by Western blotting using the Bolt system (Life Technologies). 
Cellular Target Engagement - Competition with Sulfopin-DTB.

The indicated cell lines were plated in $10 \mathrm{~cm}$ plates with 2.5 million cells per plate in $6 \mathrm{~mL}$ of media. The day after plating, cells were treated with DMSO or the indicated concentrations of candidate inhibitor for the indicated time points. The cells were then washed two times with cold PBS ( $1 \mathrm{~mL}$ per $10 \mathrm{~cm}$ plate) and collected by scraping with a cell scraper. Cells were lysed in 50 mM Hepes, pH 7.4, 1 mM EDTA, 10\% glycerol, 1 mM TCEP, 150 mM NaCl, 1 mM EDTA, 0.5\% NP-40, and protease inhibitor tablet (Roche) - using $210 \mu \mathrm{L}$ of cell lysis buffer per $10 \mathrm{~cm}$ plate of cells. After clarifying (14,000 rpm for $15 \mathrm{~min}$ ), $9 \mu \mathrm{L}$ of each lysate sample was combined with $4 \mathrm{x}$ LDS $+10 \% \beta$-mercaptoethanol (in a ratio of 3:1), boiled for 5 minutes, and set aside for the input loading control (later to be loaded directly on the gel). Then, $200 \mu \mathrm{L}$ of each lysate sample was incubated with $1 \mu \mathrm{M}$ of Sulfopin-DTB for $1 \mathrm{~h}$ at $4^{\circ} \mathrm{C}$ and processed as in "lysate pull-down with Sulfopin-DTB" (above).

\section{Radiosensitization studies}

AlamarBlue-based cell viability assays were performed as previously described ${ }^{31}$. Briefly, HeLa cells were seeded at a density of 200 cells/well in a 96-well plate. After $16 \mathrm{~h}$, cells were treated with Sulfopin and exposed to $7.5 \mathrm{~Gy}$ IR using an X-RAD 320 PRECISION X-RAY irradiator 1 hour after drug treatment. At 3 days post-IR, cells were incubated with AlamarBlue (ThermoFisher) at a final concentration of $10 \%$. At 4 days post-IR, absorbance was measured at a wavelength of $570 \mathrm{~nm}$ with a $600 \mathrm{~nm}$ reference wavelength. Relative fluorescence was calculated using cellfree wells as a control reference, and percentage survival was calculated by comparing with DMSO-treated, non-irradiated controls. Sulfopin efficacy was assessed at $24 \mathrm{~h}$ post-IR by Western blot using anti-IRAK1 (Cell Signaling Technology \#4504) and anti-IRAK1pT209 (Assay Biotech \#A1074) antibodies.

In vivo germinal centers evaluation

WT mice (C57BL/6) were provided by Harlan, Israel. All experiments with mice were approved by the Weizmann Institute IACUC committee. Mice were immunized by injection of OVA coupled to the hapten 4-hydroxy-3-nitrophenylacetyl (NP-OVA) precipitated in alum (Imject@ Alum, Thermo Scientific) into the hind footpads (25ul). Single cell suspensions were obtained by forcing popliteal lymph nodes through a $70 \mu \mathrm{m}$ mesh into ice cold FACS buffer (EDTA $1 \mathrm{mM}$ and 2\% serum in PBS). Cells were incubated with $2 \mu \mathrm{g} / \mathrm{ml}$ anti-16/32 (clone 93) for blockage of FC receptors for 5$10 \mathrm{~min}$. Cell suspensions were washed and incubated with fluorescently-labeled antibodies (B220 V500, FAS FITC, CD38 Alexa fluor 700; Biolegend) for 20-40 min. GC cells were gated as 
live/single, $\mathrm{B}_{220^{+}} \mathrm{CD} 38^{\mathrm{LO}} \mathrm{FAS}^{\mathrm{Hi}}$. Cell suspensions were analyzed by Cytoflex (Beckman) flow cytometer.

\section{Cell Viability Assays: Growth Over Time in 2D-Adherent Monolayer Cell Culture.}

The indicated cell lines were plated at a density of 500 cells per well (except PATU-8988T cells, which were plated at 100 cells per well to avoid over-confluence by day 8 ) in $100 \mu \mathrm{L}$ of media in a 96-well white clear bottom plate (Corning cat\#3903), with one plate per time point (Day 0, 2, 4, $6,8)$. Cells were treated the day after plating with $1 \mu \mathrm{L}$ of DMSO, Sulfopin, or Sulfopin-AcA to give the indicated concentrations, and were then incubated at $37^{\circ} \mathrm{C} 5 \% \mathrm{CO}_{2}$. Every $48 \mathrm{~h}$, the media was aspirated and replaced with fresh media containing fresh compound or DMSO. When the indicated time points had been reached, cell viability was evaluated using the CellTiter-Glo Luminescent Cell Viability Assay (Promega cat\#G7570) according to the manufacturer's standards, measuring luminescence using an Envision plate-reader. The Day 0 time point plates were read the day after plating, prior to compound treatment. $\mathrm{N}=3$ biological replicates were used for each treatment condition.

\section{Cell Viability Assay: 5 day treatment}

PATU-8988T cells were plated in flat bottom 96-well plates (Corning cat \#3903) at a density of 1,000 cells per well in $100 \mu \mathrm{L}$ media and were treated the next day with $1 \mu \mathrm{L}$ of the indicated compounds in a three-fold dilution series. The cells were incubated at $37^{\circ} \mathrm{C} 5 \% \mathrm{CO}_{2}$ for 5 days. Anti-proliferative effects were assessed by CellTiter-Glo Luminescent Cell Viability Assay (Promega cat \#G7570) according to the manufacturer's standards, measuring luminescence using an Envision plate-reader. $\mathrm{N}=3$ biological replicates were used for each treatment condition.

\section{Assessing Antiproliferative Activity in PATU-8988T 3D Cell Culture.}

Prepare PATU-8988T (WT or Pin $1^{-/-}$) Matrigel suspensions by resuspending cells in $100 \%$ cold Matrigel (kept on ice). Plate 1 dome per well in 24-well plate (Greiner CELLSTAR), with $50 \mu \mathrm{L}$ of cells/Matrigel suspension per dome, and 1,000 cells per dome. After plating the domes, place 24well plate on top of T175 (previously filled with autoclaved water) in the incubator, let solidify for 15 min. Keeping the 24-well plate on the T175 filled with water, move to the TC hood and carefully add $500 \mu \mathrm{L}$ of cold DMEM (+10\% FBS/1\% P/S) per well, then place 24-well plate in incubator. The next day, treat with DMSO, Sulfopin $(1 \mu \mathrm{M})$, or Sulfopin-AcA $(1 \mu \mathrm{M})$. Every 3 days, carefully aspirate off the media, add $500 \mu \mathrm{L}$ of fresh media and retreat. After 9 days, aspirate off the media, add $300 \mu \mathrm{L}$ of 3D CellTiter-Glo (Promega cat\#G9681) per well, shake plate for $1 \mathrm{~h}$. Measure 
luminescence using an Envision plate-reader. $\mathrm{N}=3$ biological replicates were used for each treatment condition.

\section{RNA sequencing}

Mino cells (acquired from the ATCC) were grown at $37^{\circ} \mathrm{C}$ in a $5 \% \mathrm{CO}_{2}$ humidified incubator and cultured in RPMI-1640 (Biological industries), supplemented with 15\% Fetal bovine serum (biological industries) and $1 \%$ pen-strep solution (biological industries). $11 \times 10^{6}$ cells were incubated with $1 \mu \mathrm{M}$ Sulfopin (0.02\% DMSO) or with $0.02 \%$ DMSO in triplicates for $6 \mathrm{~h}$. Total RNA was isolated with RNeasy kit (Qiagen). RNA libraries were prepared from $2 \mu \mathrm{g}$ total RNA using SENSE mRNA-Seq library prep kit V2 (lexogen). Total RNA and library quality was analyzed using Qubit fluorometric and TapeStation analysis (Agilent). Samples were sequenced using NextSeq 500/550 High Output Kit v2.5 (illumina) on NextSeq550.

RNA-seq reads were aligned to the human genome (hg19 assembly) using STAR ${ }^{111}$ and gene expression was determined using RSEM ${ }^{112}$ and RefSeq annotations. Differential expression was computed using DESeq $2^{113}$ with default parameters. Genes with baseMean $>50$ that were downregulated with $\mathrm{P}<0.05$ were further analysed using Enrichr ${ }^{89}$.

\section{Profiling of Sulfopin-DTB reactive cysteines by CITe-Id}

PATU-8988T cells were cultured in DMEM supplemented with 10\% Fetal Calf Serum. DMSO (Control) or Sulfopin were diluted in fresh media (final DMSO concentration $<0.1 \%$, final Sulfopin concentration: $100 \mathrm{nM}, 500 \mathrm{nM}$ and $1 \mu \mathrm{M}$ ) and added to sub-confluent cultures. After $5 \mathrm{~h}$ of incubation at $37^{\circ} \mathrm{C}$, cells were harvested using a cell scraper and centrifuged at $300 \times \mathrm{g}$ for 3 minutes at $4^{\circ} \mathrm{C}$. Cell pellets were washed with ice-cold PBS and centrifuged again. A total of 3 washes were performed before freezing cell pellets at $-80^{\circ} \mathrm{C}$. This procedure was performed twice on cells independently cultured a week apart. Frozen pellets were then processed essentially as described $^{79}$, except that pre-cleared lysates were treated with $2 \mu \mathrm{M}$ Sulfopin-DTB overnight at 4 ${ }^{\circ} \mathrm{C}$. Protein desalting, digestion, enrichment of desthiobiotin modified peptides, iTRAQ stable isotope labeling, peptide clean-up, and multidimensional LC/MS-MS analysis were then performed exactly as described ${ }^{79}$. Data processing and database search was performed as described $^{79}$, except that spectral processing accounted for Sulfopin-DTB specific fragment ions, and Sulfopin-DTB labeling of cysteine was considered as a variable modification by Mascot (version 2.6.2). Inhibitor concentrations and ratios were used to generate a trendline for each labeled site with the slope corresponding to the competitive dose response for each modified cysteine site. 
Profiling of Sulfopin reactive cysteines by rdTOP-ABPP

MDA-MB-231 cells were cultured at $37^{\circ} \mathrm{C}$ under $5 \% \mathrm{CO}_{2}$ atmosphere in DMEM culture medium supplemented with $10 \%$ FBS and $1 \%$ PS. Cells were grown to $70 \%$ confluence and incubated with DMSO or $5 \mu \mathrm{M}$ Sulfopin for $2 \mathrm{~h}$ with serum-free medium. Cells were harvested, lysed by sonication in ice-cold PBS containing $0.1 \%$ TritonX-100 and centrifuged at 100,000 $\mathrm{g}$ for $30 \mathrm{~min}$ to remove cell debris. Then protein concentrations were determined by BCA protein assay. Proteomes were normalized to $2 \mathrm{mg} / \mathrm{mL}$ in $1 \mathrm{~mL}$ for each sample.

Each of the DMSO and Sulfopin incubated proteomes was treated with $100 \mu \mathrm{M}$ iodoacetamide-alkyne (IAyne) for $1 \mathrm{~h}$ at room temperature. The proteomes were then reacted with $1 \mathrm{mM}$ CuSO4, $100 \mu \mathrm{M}$ TBTA ligand, $100 \mu \mathrm{M}$ biotin-acid-N3 tag and $1 \mathrm{mM}$ TCEP for $1 \mathrm{~h}$. After click reaction, the proteomes were centrifuged at $8000 \mathrm{~g}$ for $5 \mathrm{~min}$, then the precipitated proteins were washed for two times using cold methanol. The proteomes were resuspended in $1.2 \%$ SDS/PBS and diluted to $0.2 \%$ SDS/PBS. Finally, the samples were prepared, analyzed on LCMS/MS and quantified according to the published rdTOP-ABPP protocol ${ }^{80}$. Briefly, the beads from trypsin digestion were washed and resuspended in $100 \mu \mathrm{L}$ of TEAB buffer. $8 \mu \mathrm{L}$ of $4 \% \mathrm{D}^{13} \mathrm{CDO}$ or $\mathrm{HCHO}$ was added to the Sulfopin or DMSO sample respectively. At the same time, $8 \mu \mathrm{L}$ of 0.6 $\mathrm{M} \mathrm{NaBH}{ }_{3} \mathrm{CN}$ was added and the reaction was lasted for $2 \mathrm{~h}$ at room temperature. Then the beads were washed again and the modified peptides were cleaved by $2 \%$ formic acid. LC-MS/MS data was analyzed by ProLuCID ${ }^{114}$ with static modification of cysteine (+57.0215 Da) and variable oxidation of methionine (+15.9949 Da). The isotopic modifications (+28.0313 and +34.0631 Da for light and heavy labeling respectively) are set as static modifications on the N-terminal of a peptide and lysines. Variable modification on cysteines is set at $+322.23688 \mathrm{Da}$. The ratios were quantified by the CIMAGE software ${ }^{115}$.

\section{Myc luciferase reporter assay}

HEK-293 cells were maintained in DMEM supplemented with $10 \%$ standard fetal bovine serum (FBS), $2.5 \mathrm{mM} \mathrm{L-glutamine,} \mathrm{NEEA} \mathrm{and} \mathrm{1x} \mathrm{penicillin/streptomycin.} \mathrm{Cells} \mathrm{were} \mathrm{passaged} \mathrm{to} 80 \%$ confluence in 6-well plates and transfected with 4x-Ebox-Luc and Pin1-Flag plasmids as indicated and $\beta$-galactosidase as an internal control ${ }^{22}$ using Lipofectamine 3000 (Invitrogen, Carlsbad, CA) following manufacturer's protocol. Sulfopin treatments were performed at the time of transfection and cells were harvested $48 \mathrm{~h}$ later.

Cell were washed with PBS, and then lysed in 1X cell lysis buffer (Promega, Madison, $\mathrm{WI}$ ). Lysates were sonicated for 10 pulses at an output $=1$ and $10 \%$ duty (Branson), and incubated on ice for $20 \mathrm{~min}$. Lysates were then cleared by centrifugation at $14 \mathrm{~K} \mathrm{rpm}$ for $10 \mathrm{~min}$ at $4^{\circ} \mathrm{C}$. 
Luciferase activity was measured using the Promega Luciferase Assay Kit and Berthold luminometer (Bundoora, Australia) and normalized to $\beta$-galactosidase activity ${ }^{116}$.

\section{Zebrafish Neuroblastoma Models}

All zebrafish studies and maintenance of the animals were performed in accordance with DanaFarber Cancer Institute IACUC-approved protocol \#02-107. For in vivo drug treatment, 3-day-old zebrafish embryos were placed in 48-well plates with 5 embryos per well, and treated with DMSO control or Sulfopin in standard egg water.

For neuroblastoma transplantation, zebrafish neuroblastoma cells were harvested by dicing 4-month-old Tg(dßh:MYCN;dßh:EGFP) transgenic zebrafish in PBS. The cell suspension was filtered with Falcon 40- $\mu$ m cell strainer (Corning, Corning, NY, USA) and loaded into thin-wall borosilicate glass capillary needles $(1.0 \mathrm{~mm}$ OD, $0.75 \mathrm{~mm}$ ID; World Precision Instruments, Sarasota, FL, USA). The recipients, 2-day-old zebrafish Casper embryos, were manually dechorionized and anaesthetized with $0.003 \%$ tricaine (Sigma) before being positioned on a 10 $\mathrm{cm}$ Petri dish coated with $1 \%$ agarose. Intravenous tumor transplantation was performed as described $^{94}$, with 200 400 cells injected into the Duct of Cuvier of each recipient. One day later, the 3-day-old recipients were randomly divided into 48-well plates and treated with DMSO control or Sulfopin in standard egg water for five days, with drug refreshment on the second day of the treatment.

A Nikon SMZ1500 microscope equipped with a Nikon digital sight DS-U1 camera was used for capturing both the bright field and fluorescent images from live zebrafish and embryos. For PSNS and neuroblastoma quantification, all animals in the same experiments were imaged under the same conditions and the acquired fluorescent images were quantified using ImageJ software by measuring the area of the EGFP fluorescent tumor mass.

\section{Mice studies (PK/PD/Tox)}

PK data was obtained as fee-for-service from Scripps Florida. For the toxicity study 18 mice were used for the following arms: 3 control mice injected with vehicle, 3 mice $10 \mathrm{mg} / \mathrm{kg}$ Sulfopin injected once daily, 3 mice $20 \mathrm{mg} / \mathrm{kg}$ Sulfopin injected once daily, $3 \mathrm{mice} 20 \mathrm{mg} / \mathrm{kg}$ Sulfopin injected once every other day, 3 mice $40 \mathrm{mg} / \mathrm{kg}$ Sulfopin injected once daily, 3 mice $40 \mathrm{mg} / \mathrm{kg}$ Sulfopin injected once every other day. Formulation was ( $5 \%$ NMP, $5 \%$ solutol, $20 \%$ DMSO). Mice were treated for 14 days.

PD Study was performed at the Dana-Farber Cancer Institute, and the procedure was approved by IACUC under protocol 16-015. Mice were treated for 3 total doses spanning 2 
consecutive days with vehicle, or Sulfopin (10 or $20 \mathrm{mpk}$ ) by oral gavage, after which the organs were harvested $4 \mathrm{~h}$ after the last dose. The spleen of each mouse was ground and lysed in 300 $\mu \mathrm{L}$ of $50 \mathrm{mM}$ Hepes, pH 7.4, 1 mM EDTA, 10\% glycerol, 1 mM TCEP, 150 mM NaCl, 1 mM EDTA, $0.5 \%$ NP-40, and protease inhibitor tablet (Roche). After clarifying (14,000 rpm for $15 \mathrm{~min}$ ), the lysates were normalized by BCA and diluted to a final concentration of $2.5 \mu \mathrm{g} / \mu \mathrm{L} .200 \mu \mathrm{L}$ of 2.5 $\mu \mathrm{g} / \mu \mathrm{L}$ of each spleen sample was then incubated with $1 \mu \mathrm{M}$ of Sulfopin-DTB for $1 \mathrm{~h}$ at $4^{\circ} \mathrm{C}$ and processed as in "lysate pull-down with Sulfopin-DTB" (above). To prepare the input samples, each original lysate sample (prior to pull-down) was combined with 4x LDS + 10\% $\beta$-mercaptoethanol (in a ratio of 3:1), boiled for 5 minutes, and $25 \mu \mathrm{g}$ of each sample was then loaded on the gel.

\section{Murine Neuroblastoma models}

All experiments were approved by The Institute of Cancer Research Animal Welfare and Ethical Review Body and performed in accordance with the UK Home Office Animals (Scientific Procedures) Act 1986, the United Kingdom National Cancer Research Institute guidelines for the welfare of animals in cancer research ${ }^{117}$ and the ARRIVE (animal research: reporting in vivo experiments) guidelines ${ }^{118}$.

Transgenic Th-MYCN mice were genotyped to detect the presence of the human MYCN transgene ${ }^{119}$. The study was performed using both male and female hemizygous mice, which developed palpable tumors at 50 to 130 days with a $25 \%$ penetrance. Tumor development was monitored weekly by palpation by an experienced animal technician. Mice with palpable tumors at $>/=4-5 \mathrm{~mm}$ were then enrolled into two groups. Group 1: Animals will all received Sulfopin at $40 \mathrm{mg} / \mathrm{kg}$, once per day by oral gavage. Group 2: Animals will all received Vehicle (5\% NMP, 5\% kolliphor, 20\% DMSO) once per day by oral gavage. Studies were terminated when the mean diameter of the tumor reached $15 \mathrm{~mm}$. Tumor volumes were measured by Vernier caliper across two perpendicular diameters, and volumes were calculated according to the formula: $V=4 / 3 \pi$ $[(d 1+d 2) / 4]^{3}$. Mice were housed in specific pathogen-free rooms in autoclaved, aseptic microisolator cages (maximum of four mice per cage). 


\section{Acknowledgments}

N.L. is the incumbent of the Alan and Laraine Fischer Career Development Chair; N.L. would like to acknowledge funding from the Israel Science Foundation (grant No. 2462/19), The Rising Tide Foundation, The Israel Cancer Research Fund, the Israeli Ministry of Science. Technology (grant No. 3-14763), and the Moross integrated cancer center. N.L. is also supported by the Helen and Martin Kimmel Center for Molecular Design, Joel and Mady Dukler Fund for Cancer Research, the Estate of Emile Mimran and Virgin JustGiving and the George Schwartzman Fund. C.D. was supported by the Minerva Fellowship program of the Max Planck Society, funded by the German Federal Ministry for Education and Research. The work was supported in part by NIH grant R01CA205153 to K.P.L., N. S. G. and X. Z. Z. N.S.G. was also supported by the Hale Center for Pancreatic Research. Y.C. \& C.W. thank the Computing Platform of the Center for Life Science at Peking University for supporting the proteomic data analysis. S.D.P acknowledges funding from the Linde Family Foundation. Part of this research was conducted at the Advanced Photon Source on the Northeastern Collaborative Access Team beamlines (NIGMS P41 GM103403) and SBGrid compiled software (eLife 2013;2:e01456). J.A.M. acknowledges support from the National Institutes of Health (CA233800) and the Mark Foundation for Cancer Research. B.J.P. was supported by the Ruth L. Kirschstein NRSA Individual Predoctoral Fellowship (F31 CA225066), the Training Grant in Chemical Biology (NIH 5 T32 GM007306-41), the Training Grant in Pharmacological Sciences (NIH 5 T32 GM095450-04, B.J.P.), and the Chleck Foundation (B.J.P./Z.M.D.). B.N. was supported by an American Cancer Society Postdoctoral Fellowship (PF17-010-01-CDD) and the Katherine L. and Steven C. Pinard Research Fund (N.S.G. and B.N.). L. C. is supported by the Cancer Research UK Program Grant (C34648/A18339 and C34648/A14610). Y. J. is supported by a Children with Cancer UK Research Fellowship (2014/176). R.C.S. acknowledges funding support from NCI R01s CA196228 and CA186241 and foundation support from The Brenden-Colson Center for Pancreatic Care. We thank Igor Ulitski for help with RNA-seq analysis and Milka Kostic for critical reading of the manuscript. 


\section{Conflict of Interests}

N.S.G. is a Scientific Founder and member of the Scientific Advisory Board (SAB) of C4, 506 Therapeutics, Syros, Soltego, Gatekeeper and Petra Pharmaceuticals and has received research funding from Novartis, Astellas, Taiho and Deerfield. N.L. is a member of the SAB of Trilogy Sciences, and has received research support from Teva. J.A.M. has received support through sponsored research agreements with AstraZeneca and Vertex. J.A.M. serves on the SAB of 908 Devices. C.M.B. is an employee of AstraZeneca. C.D., B.J.P., D.Z., S.H., X.L., K.P.L., X.Z.Z., A.T.L., N.S.G. and N.L. are inventors on a patent application related to the inhibitors described in this manuscript. 


\section{References}

1. Hanahan, D. \& Weinberg, R. A. Hallmarks of cancer: the next generation. Cell 144, 646$674(2011)$.

2. Pawson, T. \& Scott, J. D. Protein phosphorylation in signaling--50 years and counting.

Trends Biochem. Sci. 30, 286-290 (2005).

3. Blume-Jensen, P. \& Hunter, T. Oncogenic kinase signalling. Nature 411, 355-365 (2001).

4. Lu, K. P. \& Zhou, X. Z. The prolyl isomerase PIN1: a pivotal new twist in phosphorylation signalling and disease. Nat. Rev. Mol. Cell Biol. 8, 904-916 (2007).

5. Lam, P. B. et al. Prolyl isomerase Pin1 is highly expressed in Her2-positive breast cancer and regulates erbB2 protein stability. Mol. Cancer 7, 91 (2008).

6. Liao, Y. et al. Peptidyl-prolyl cis/trans isomerase Pin1 is critical for the regulation of PKB/Akt stability and activation phosphorylation. Oncogene 28, 2436-2445 (2009).

7. Lee, T. H. et al. Essential role of Pin1 in the regulation of TRF1 stability and telomere maintenance. Nat. Cell Biol. 11, 97-105 (2009).

8. Ryo, A., Nakamura, M., Wulf, G., Liou, Y. C. \& Lu, K. P. Pin1 regulates turnover and subcellular localization of beta-catenin by inhibiting its interaction with APC. Nat. Cell Biol. 3, 793-801 (2001).

9. Ryo, A. et al. Regulation of NF-kappaB signaling by Pin1-dependent prolyl isomerization and ubiquitin-mediated proteolysis of p65/RelA. Mol. Cell 12, 1413-1426 (2003).

10. Shen, M., Stukenberg, P. T., Kirschner, M. W. \& Lu, K. P. The essential mitotic peptidylprolyl isomerase Pin1 binds and regulates mitosis-specific phosphoproteins. Genes Dev. 12, 706-720 (1998).

11. Stukenberg, P. T. \& Kirschner, M. W. Pin1 acts catalytically to promote a conformational change in Cdc25. Mol. Cell 7, 1071-1083 (2001).

12. Xiang, K. et al. Crystal structure of the human symplekin-Ssu72-CTD phosphopeptide complex. Nature 467, 729-733 (2010). 
13. Zhou, X. Z. et al. Pin1-dependent prolyl isomerization regulates dephosphorylation of Cdc25C and tau proteins. Mol. Cell 6, 873-883 (2000).

14. Brown, N. R., Noble, M. E., Endicott, J. A. \& Johnson, L. N. The structural basis for specificity of substrate and recruitment peptides for cyclin-dependent kinases. Nat. Cell Biol. 1, 438-443 (1999).

15. Chen, Y. et al. Prolyl isomerase Pin1: a promoter of cancer and a target for therapy. Cell Death Dis. 9, 883 (2018).

16. Zhou, X. Z. \& Lu, K. P. The isomerase PIN1 controls numerous cancer-driving pathways and is a unique drug target. Nat. Rev. Cancer 16, 463-478 (2016).

17. Bao, L. et al. Prevalent overexpression of prolyl isomerase Pin1 in human cancers. Am. J. Pathol. 164, 1727-1737 (2004).

18. Lu, K. P. Prolyl isomerase Pin1 as a molecular target for cancer diagnostics and therapeutics. Cancer Cell 4, 175-180 (2003).

19. Tan, X. et al. Pin1 expression contributes to lung cancer: Prognosis and carcinogenesis. Cancer Biol. Ther. 9, 111-119 (2010).

20. Li, Q. et al. The rs2233678 polymorphism in PIN1 promoter region reduced cancer risk: a meta-analysis. PLoS One 8, e68148 (2013).

21. Rustighi, A. et al. The prolyl-isomerase Pin1 is a Notch1 target that enhances Notch1 activation in cancer. Nature Cell Biology vol. 11 133-142 (2009).

22. Farrell, A. S. et al. Pin1 regulates the dynamics of c-Myc DNA binding to facilitate target gene regulation and oncogenesis. Mol. Cell. Biol. 33, 2930-2949 (2013).

23. Brenkman, A. B. et al. The peptidyl-isomerase Pin1 regulates p27kip1 expression through inhibition of Forkhead box O tumor suppressors. Cancer Res. 68, 7597-7605 (2008).

24. Basu, A. et al. Proteasomal degradation of human peptidyl prolyl isomerase pin1-pointing phospho Bcl2 toward dephosphorylation. Neoplasia 4, 218-227 (2002).

25. Boldetti, A., Guarnaccia, V., Rambaldi, A. \& Parrella, E. Inhibition of the peptidyl-prolyl- 
isomerase Pin1 enhances the responses of acute myeloid leukemia cells to retinoic acid via stabilization of RAR a and PML-RARa. Cancer Res. (2009).

26. Girardini, J. E. et al. A Pin1/Mutant p53 Axis Promotes Aggressiveness in Breast Cancer. Cancer Cell vol. 20 79-91 (2011).

27. Wulf, G., Garg, P., Liou, Y.-C., Iglehart, D. \& Lu, K. P. Modeling breast cancer in vivo and ex vivo reveals an essential role of Pin1 in tumorigenesis. EMBO J. 23, 3397-3407 (2004).

28. D'Artista, L. et al. Pin1 is required for sustained B cell proliferation upon oncogenic activation of Myc. Oncotarget 7, 21786-21798 (2016).

29. Zheng, M. et al. Inhibition of the prolyl isomerase Pin1 enhances the ability of sorafenib to induce cell death and inhibit tumor growth in hepatocellular carcinoma. Oncotarget 8, 29771-29784 (2017).

30. Ding, Q. et al. Down-regulation of myeloid cell leukemia-1 through inhibiting Erk/Pin 1 pathway by sorafenib facilitates chemosensitization in breast cancer. Cancer Res. 68, 6109-6117 (2008).

31. Liu, P. H. et al. An IRAK1-PIN1 signalling axis drives intrinsic tumour resistance to radiation therapy. Nat. Cell Biol. 21, 203-213 (2019).

32. Luo, M.-L. et al. The Rab2A GTPase promotes breast cancer stem cells and tumorigenesis via Erk signaling activation. Cell Rep. 11, 111-124 (2015).

33. Rustighi, A. et al. Prolyl-isomerase Pin1 controls normal and cancer stem cells of the breast. EMBO Mol. Med. 6, 99-119 (2014).

34. Tsherniak, A. et al. Defining a Cancer Dependency Map. Cell 170, 564-576.e16 (2017).

35. Liou, Y.-C. et al. Loss of Pin1 function in the mouse causes phenotypes resembling cyclin D1-null phenotypes. Proceedings of the National Academy of Sciences vol. 99 1335-1340 (2002).

36. Hennig, L. et al. Selective inactivation of parvulin-like peptidyl-prolyl cis/trans isomerases by juglone. Biochemistry 37, 5953-5960 (1998). 
37. Wei, S. et al. Active Pin1 is a key target of all-trans retinoic acid in acute promyelocytic leukemia and breast cancer. Nat. Med. 21, 457-466 (2015).

38. Kozono, S. et al. Arsenic targets Pin1 and cooperates with retinoic acid to inhibit cancerdriving pathways and tumor-initiating cells. Nat. Commun. 9, 3069 (2018).

39. Campaner, E. et al. A covalent PIN1 inhibitor selectively targets cancer cells by a dual mechanism of action. Nat. Commun. 8, 15772 (2017).

40. Lu, Z. \& Hunter, T. Prolyl isomerase Pin1 in cancer. Cell Res. 24, 1033-1049 (2014).

41. Moore, J. D. \& Potter, A. Pin1 inhibitors: Pitfalls, progress and cellular pharmacology. Bioorg. Med. Chem. Lett. 23, 4283-4291 (2013).

42. Fila, C., Metz, C. \& van der Sluijs, P. Juglone inactivates cysteine-rich proteins required for progression through mitosis. J. Biol. Chem. 283, 21714-21724 (2008).

43. Pinch, B. J. et al. Discovery and Characterization of a Potent and Selective Pin1 Inhibitor Targeted to an Active Site Cysteine. Nat. Chem. Biol. (2020) doi:In press.

44. Baillie, T. A. Targeted Covalent Inhibitors for Drug Design. Angew. Chem. Int. Ed Engl. 55, 13408-13421 (2016).

45. Noe, M. C. \& Gilbert, A. M. Targeted covalent enzyme inhibitors. in Annual Reports in Medicinal Chemistry vol. 47 413-439 (Elsevier, 2012).

46. Johnson, D. S., Weerapana, E. \& Cravatt, B. F. Strategies for discovering and derisking covalent, irreversible enzyme inhibitors. Future Med. Chem. 2, 949-964 (2010).

47. Ghosh, A. K., Samanta, I., Mondal, A. \& Liu, W. R. Covalent Inhibition in Drug Discovery. ChemMedChem 14, 889-906 (2019).

48. Singh, J., Petter, R. C., Baillie, T. A. \& Whitty, A. The resurgence of covalent drugs. Nat. Rev. Drug Discov. 10, 307-317 (2011).

49. Cross, D. A. E. et al. AZD9291, an irreversible EGFR TKI, overcomes T790M-mediated resistance to EGFR inhibitors in lung cancer. Cancer Discov. 4, 1046-1061 (2014).

50. Ward, R. A. et al. Structure- and reactivity-based development of covalent inhibitors of the 
activating and gatekeeper mutant forms of the epidermal growth factor receptor (EGFR). J. Med. Chem. 56, 7025-7048 (2013).

51. Bauer, R. A. Covalent inhibitors in drug discovery: from accidental discoveries to avoided liabilities and designed therapies. Drug Discov. Today 20, 1061-1073 (2015).

52. Ostrem, J. M., Peters, U., Sos, M. L., Wells, J. A. \& Shokat, K. M. K-Ras(G12C) inhibitors allosterically control GTP affinity and effector interactions. Nature 503, 548-551 (2013).

53. Patricelli, M. P. et al. Selective Inhibition of Oncogenic KRAS Output with Small Molecules Targeting the Inactive State. Cancer Discov. 6, 316-329 (2016).

54. Janes, M. R. et al. Targeting KRAS Mutant Cancers with a Covalent G12C-Specific Inhibitor. Cell 172, 578-589.e17 (2018).

55. Shin, Y. et al. Discovery of N-(1-Acryloylazetidin-3-yl)-2-(1H-indol-1-yl)acetamides as Covalent Inhibitors of KRASG12C. ACS Med. Chem. Lett. 10, 1302-1308 (2019).

56. Leach, A. R., Hann, M. M., Burrows, J. N. \& Griffen, E. J. Fragment screening: an introduction. Molecular BioSystems vol. 2429 (2006).

57. Baker, M. Fragment-based lead discovery grows up. Nat. Rev. Drug Discov. 12, 5-7 (2013).

58. Erlanson, D. A., McDowell, R. S. \& O’Brien, T. Fragment-based drug discovery. J. Med. Chem. 47, 3463-3482 (2004).

59. Scott, D. E., Coyne, A. G., Hudson, S. A. \& Abell, C. Fragment-based approaches in drug discovery and chemical biology. Biochemistry 51, 4990-5003 (2012).

60. Hall, R. J., Mortenson, P. N. \& Murray, C. W. Efficient exploration of chemical space by fragment-based screening. Prog. Biophys. Mol. Biol. 116, 82-91 (2014).

61. Hann, M. M., Leach, A. R. \& Harper, G. Molecular complexity and its impact on the probability of finding leads for drug discovery. J. Chem. Inf. Comput. Sci. 41, 856-864 (2001).

62. Resnick, E. et al. Rapid Covalent-Probe Discovery by Electrophile-Fragment Screening. J. 
Am. Chem. Soc. 141, 8951-8968 (2019).

63. Erlanson, D. A. et al. Site-directed ligand discovery. Proceedings of the National Academy of Sciences vol. 97 9367-9372 (2000).

64. Erlanson, D. A., Wells, J. A. \& Braisted, A. C. Tethering: Fragment-Based Drug Discovery. Annual Review of Biophysics and Biomolecular Structure vol. 33 199-223 (2004).

65. Johansson, H. et al. Fragment-Based Covalent Ligand Screening Enables Rapid Discovery of Inhibitors for the RBR E3 Ubiquitin Ligase HOIP. J. Am. Chem. Soc. 141, 2703-2712 (2019).

66. Kathman, S. G., Xu, Z. \& Statsyuk, A. V. A fragment-based method to discover irreversible covalent inhibitors of cysteine proteases. J. Med. Chem. 57, 4969-4974 (2014).

67. Backus, K. M. et al. Proteome-wide covalent ligand discovery in native biological systems. Nature 534, 570-574 (2016).

68. Parker, C. G. et al. Ligand and Target Discovery by Fragment-Based Screening in Human Cells. Cell 168, 527-541.e29 (2017).

69. Roberts, A. M. et al. Chemoproteomic Screening of Covalent Ligands Reveals UBA5 As a Novel Pancreatic Cancer Target. ACS Chem. Biol. 12, 899-904 (2017).

70. Grossman, E. A. et al. Covalent Ligand Discovery against Druggable Hotspots Targeted by Anti-cancer Natural Products. Cell Chem Biol 24, 1368-1376.e4 (2017).

71. Counihan, J. L., Wiggenhorn, A. L., Anderson, K. E. \& Nomura, D. K. ChemoproteomicsEnabled Covalent Ligand Screening Reveals ALDH3A1 as a Lung Cancer Therapy Target. ACS Chem. Biol. 13, 1970-1977 (2018).

72. Anderson, K. E., To, M., Olzmann, J. A. \& Nomura, D. K. Chemoproteomics-Enabled Covalent Ligand Screening Reveals a Thioredoxin-Caspase 3 Interaction Disruptor That Impairs Breast Cancer Pathogenicity. ACS Chemical Biology vol. 12 2522-2528 (2017).

73. London, N. et al. Covalent docking of large libraries for the discovery of chemical probes. Nat. Chem. Biol. 10, 1066-1072 (2014). 
74. Zhang, Y. et al. Structural basis for high-affinity peptide inhibition of human Pin1. ACS Chem. Biol. 2, 320-328 (2007).

75. Yaffe, M. B. et al. Sequence-specific and phosphorylation-dependent proline isomerization: a potential mitotic regulatory mechanism. Science 278, 1957-1960 (1997).

76. Guo, C. et al. Structure-based design of novel human Pin1 inhibitors (I). Bioorg. Med. Chem. Lett. 19, 5613-5616 (2009).

77. Dong, L. et al. Structure-based design of novel human Pin1 inhibitors (II). Bioorg. Med. Chem. Lett. 20, 2210-2214 (2010).

78. Guo, C. et al. Structure-based design of novel human Pin1 inhibitors (III): optimizing affinity beyond the phosphate recognition pocket. Bioorg. Med. Chem. Lett. 24, 4187-4191 (2014).

79. Browne, C. M. et al. A Chemoproteomic Strategy for Direct and Proteome-Wide Covalent Inhibitor Target-Site Identification. J. Am. Chem. Soc. 141, 191-203 (2019).

80. Yang, F., Gao, J., Che, J., Jia, G. \& Wang, C. A Dimethyl-Labeling-Based Strategy for SiteSpecifically Quantitative Chemical Proteomics. Anal. Chem. 90, 9576-9582 (2018).

81. Phan, R. T., Saito, M., Kitagawa, Y., Means, A. R. \& Dalla-Favera, R. Genotoxic stress regulates expression of the proto-oncogene Bcl6 in germinal center B cells. Nat. Immunol. 8, 1132-1139 (2007).

82. Yu, C. et al. High-throughput identification of genotype-specific cancer vulnerabilities in mixtures of barcoded tumor cell lines. Nat. Biotechnol. 34, 419-423 (2016).

83. Chao, S. H., Greenleaf, A. L. \& Price, D. H. Juglone, an inhibitor of the peptidyl-prolyl isomerase Pin1, also directly blocks transcription. Nucleic Acids Res. 29, 767-773 (2001).

84. Baker, L. A., Tiriac, H., Clevers, H. \& Tuveson, D. A. Modeling pancreatic cancer with organoids. Trends Cancer Res. 2, 176-190 (2016).

85. Pelengaris, S., Khan, M. \& Evan, G. c-MYC: more than just a matter of life and death. Nat. Rev. Cancer 2, 764-776 (2002).

86. Yeh, E. et al. A signalling pathway controlling c-Myc degradation that impacts oncogenic 
transformation of human cells. Nat. Cell Biol. 6, 308-318 (2004).

87. Su, Y. et al. Post-translational modification localizes MYC to the nuclear pore basket to regulate a subset of target genes involved in cellular responses to environmental signals. Genes Dev. 32, 1398-1419 (2018).

88. Helander, S. et al. Pre-Anchoring of Pin1 to Unphosphorylated c-Myc in a Fuzzy Complex Regulates c-Myc Activity. Structure 23, 2267-2279 (2015).

89. Kuleshov, M. V. et al. Enrichr: a comprehensive gene set enrichment analysis web server 2016 update. Nucleic Acids Res. 44, W90-7 (2016).

90. Zhu, S. et al. Activated ALK collaborates with MYCN in neuroblastoma pathogenesis. Cancer Cell 21, 362-373 (2012).

91. He, S. et al. Synergy between loss of NF1 and overexpression of MYCN in neuroblastoma is mediated by the GAP-related domain. Elife 5, (2016).

92. Zimmerman, M. W. et al. MYC Drives a Subset of High-Risk Pediatric Neuroblastomas and Is Activated through Mechanisms Including Enhancer Hijacking and Focal Enhancer Amplification. Cancer Discov. 8, 320-335 (2018).

93. Campbell, K. et al. Association of MYCN copy number with clinical features, tumor biology, and outcomes in neuroblastoma: A report from the Children's Oncology Group. Cancer 123, 4224-4235 (2017).

94. He, S. et al. Neutrophil-mediated experimental metastasis is enhanced by VEGFR inhibition in a zebrafish xenograft model. J. Pathol. 227, 431-445 (2012).

95. Chesler, L. \& Weiss, W. A. Genetically engineered murine models - Contribution to our understanding of the genetics, molecular pathology and therapeutic targeting of neuroblastoma. Seminars in Cancer Biology vol. 21 245-255 (2011).

96. Lanning, B. R. et al. A road map to evaluate the proteome-wide selectivity of covalent kinase inhibitors. Nat. Chem. Biol. 10, 760-767 (2014).

97. Uchida, T. et al. Pin1 and Par14 peptidyl prolyl isomerase inhibitors block cell proliferation. 
Chem. Biol. 10, 15-24 (2003).

98. Tatara, Y., Lin, Y.-C., Bamba, Y., Mori, T. \& Uchida, T. Dipentamethylene thiuram monosulfide is a novel inhibitor of Pin1. Biochem. Biophys. Res. Commun. 384, 394-398 (2009).

99. Yoon, H.-E. et al. Inhibition of Plk1 and Pin1 by 5'-nitro-indirubinoxime suppresses human lung cancer cells. Cancer Lett. 316, 97-104 (2012).

100.Subedi, A. et al. Discovery of novel selenium derivatives as Pin1 inhibitors by highthroughput screening. Biochem. Biophys. Res. Commun. 474, 528-533 (2016).

101.Lee, N. Y. et al. The prolyl isomerase Pin1 interacts with a ribosomal protein S6 kinase to enhance insulin-induced AP-1 activity and cellular transformation. Carcinogenesis 30, 671681 (2009).

102.Kanaoka, R. et al. Pin1 Inhibitor Juglone Exerts Anti-Oncogenic Effects on LNCaP and DU145 Cells despite the Patterns of Gene Regulation by Pin1 Differing between These Cell Lines. PLoS One 10, e0127467 (2015).

103.Fan, G. et al. Peptidyl-prolyl isomerase Pin1 markedly enhances the oncogenic activity of the rel proteins in the nuclear factor-kappaB family. Cancer Res. 69, 4589-4597 (2009).

104.Cole, K. A. et al. RNAi screen of the protein kinome identifies checkpoint kinase 1 (CHK1) as a therapeutic target in neuroblastoma. Proc. Natl. Acad. Sci. U. S. A. 108, 3336-3341 (2011).

105. Hogarty, M. D. \& Maris, J. M. PI3King on MYCN to improve neuroblastoma therapeutics. Cancer cell vol. 21 145-147 (2012).

106.Kabsch, W. XDS. Acta Crystallogr. D Biol. Crystallogr. 66, 125-132 (2010).

107.McCoy, A. J. et al. Phaser crystallographic software. J. Appl. Crystallogr. 40, 658-674 (2007).

108.Adams, P. D. et al. PHENIX: a comprehensive Python-based system for macromolecular structure solution. Acta Crystallogr. D Biol. Crystallogr. 66, 213-221 (2010). 
109.Emsley, P. \& Cowtan, K. Coot: model-building tools for molecular graphics. Acta Crystallogr. D Biol. Crystallogr. 60, 2126-2132 (2004).

110.Auld, D. S. et al. Receptor Binding Assays for HTS and Drug Discovery. in Assay Guidance Manual (eds. Sittampalam, G. S. et al.) (Eli Lilly \& Company and the National Center for AdvancingTranslational Sciences, 2012).

111.Dobin, A. et al. STAR: ultrafast universal RNA-seq aligner. Bioinformatics 29, 15-21 (2013).

112.Li, B. \& Dewey, C. N. RSEM: accurate transcript quantification from RNA-Seq data with or without a reference genome. BMC Bioinformatics 12, 323 (2011).

113.Love, M. I., Huber, W. \& Anders, S. Moderated estimation of fold change and dispersion for RNA-seq data with DESeq2. Genome Biol. 15, 550 (2014).

114.Xu, T. et al. ProLuCID: An improved SEQUEST-like algorithm with enhanced sensitivity and specificity. J. Proteomics 129, 16-24 (2015).

115.Weerapana, E. et al. Quantitative reactivity profiling predicts functional cysteines in proteomes. Nature 468, 790-795 (2010).

116.Sears, R., Ohtani, K. \& Nevins, J. R. Identification of positively and negatively acting elements regulating expression of the E2F2 gene in response to cell growth signals. Mol. Cell. Biol. 17, 5227-5235 (1997).

117.Workman, P. et al. Guidelines for the welfare and use of animals in cancer research. British Journal of Cancer vol. 102 1555-1577 (2010).

118.Kilkenny, C., Browne, W. J., Cuthill, I. C., Emerson, M. \& Altman, D. G. Improving bioscience research reporting: The ARRIVE guidelines for reporting animal research. J. Pharmacol. Pharmacother. 1, 94-99 (2010).

119.Weiss, W. A., Aldape, K., Mohapatra, G., Feuerstein, B. G. \& Bishop, J. M. Targeted expression of MYCN causes neuroblastoma in transgenic mice. EMBO J. 16, 2985-2995 (1997). 